Open Access

\title{
Biquaternion beamspace with its application to vector-sensor array direction findings and polarization estimations
}

\author{
Dan Li, Feng Xu, Jing Fei Jiang and Jian Qiu Zhang* (D)
}

\begin{abstract}
In this paper, a biquaternion beamspace, constructed by projecting the original data of an electromagnetic vector-sensor array into a subspace of a lower dimension via a quaternion transformation matrix, is first proposed. To estimate the direction and polarization angles of sources, biquaternion beamspace multiple signal classification (BB-MUSIC) estimators are then formulated. The analytical results show that the biquaternion beamspaces offer us some additional degrees of freedom to simultaneously achieve three goals. One is to save the memory spaces for storing the data covariance matrix and reduce the computation efforts of the eigen-decomposition. Another is to decouple the estimations of the sources' polarization parameters from those of their direction angles. The other is to blindly whiten the coherent noise of the six constituent antennas in each vector-sensor. It is also shown that the existing biquaternion multiple signal classification (BQ-MUSIC) estimator is a specific case of our BB-MUSIC ones. The simulation results verify the correctness and effectiveness of the analytical ones.
\end{abstract}

Keywords: Array signal processing, Biquaternion beamspace, Biquaternion beamspace music, Direction of arrival estimation, Polarization parameters estimation

\section{Introduction}

An electromagnetic (EM) vector-sensor, which consists of six spatially collocated antennas, measures the complete electric and magnetic fields induced by EM signals [1]. Except for spatially and temporally sampling the incident EM source signals as done by a scalar-senor array $[2,3]$, an EM vector-sensor array can also record the source direction information by the signal responses of the six constituent antennas in each vector-sensor (see the $V\left(\theta_{k}, \phi_{k}\right)$ item in (2)). Moreover, an EM vector-sensor array-manifold is also sensitive to incident wavefields' polarization states [4-6], which is useful in applications, for example remote sensing [7]. It has been understood that an EM vector-sensor array can record more source information than the scalar-sensor one. However, its signal processing techniques are also more complex than the scalar-sensor ones for at least two reasons. One is that the number of antennas in a vector-sensor array is

*Correspondence: jqzhang01@fudan.edu.cn

Research Center of Smart Networks and Systems, The Department of

Electronic Engineering, Fudan University, Handan Road, Shanghai, China six times more than that in a scalar-sensor array with the same array aperture. It means that more computation efforts and memory spaces are required for vector-sensor array signal processing. Another is that a computation prohibitive four-dimensional (4D) search over the direction and polarization parameters is required [5], when the traditional multiple signal classification (MUSIC) algorithm for scalar-sensor array processing $[2,3]$ is directly extended to the vector one. Thus, it is strongly desirable to find some fast and computation effective techniques for vector-sensor array signal processing.

To achieve a significant reduction in computation time, many efforts have been made. For example, the beamspace approaches have been proposed in [8-10] for a scalarsensor array processing case, in which the original sensor data are projected into a subspace of a lower dimension (i.e., the beamspace) to reduce the data size. In the vector-sensor case, Wong and Zoltowski in [5] projected the array data into a spatio-polarizational beamspace to decouple the polarization estimation from the direction finding. With their techniques, only a 2D search is needed 
for estimating the sources' direction angles before their polarization parameters are estimated.

However, their beamspace for each source has to be designed separately to decouple the polarization estimation from direction finding.

Then a promising alternative for reducing the computation costs of the vector-sensor array processing is the using of the quaternion and biquaternion algebra. Miron et al. in [11] proposed a quaternion MUSIC (Q-MUSIC) estimator for two-component vector-sensor array processing. Zhang et al. in [12] improved the performance of the Q-MUSIC estimator for colored noise, but only for a two-component vector-sensor array. Miron's research was later extended to a three-component vector-sensor array via the biquaternion algebra [13, 14], where a biquaternion version MUSIC (BQ-MUSIC) estimator was introduced. Gong et al. in [15] improved its robustness to colored noise by diagonalizing the biquaternion covariance matrix. Using the calculation rules of the quaternion and biquaternion algebras, the computation efforts and memory spaces of their covariance matrix are significantly reduced. To handle a six-component EM vector-sensor array, Gong et al. in [16] proposed a quad-quaternion MUSIC (QQ-MUSIC) estimator. Although it was shown in [13] by Monte Carlo simulations that the BQ-MUSIC estimator was robust to the coherent corrupting noise and polarization errors, it was kept unknown what kind of reasons were for the robustness. Furthermore, the BQMUSIC was suitable for the direction finding of sources with known polarization parameters. Unfortunately, both the sources' directions and polarization angles are normally unknown in applications. The QQ-MUSIC estimator in [16] cannot also be used to estimate the polarization angles of the sources. The geometric algebra model (GMODEL) of an EM vector-sensor array is given in [17]. It is, however, kept unknown how to use it for array processing applications. On the other hand, The biquaternion Capon beamformer was proposed in [18] and [19], but they cannot estimate the polarization angles of a source.

In this paper, the techniques of projecting the output data of an electromagnetic vector-sensor array into a biquaternion beamspace via a quaternion beamformer (or a quaternion transformation matrix) are first proposed. The general form of the biquaternion beamspace multiple signal classification (BB-MUSIC) estimator is formulated by using the eigen-decomposition (EVD) of the biquaternion matrix $[13,20]$. In our biquaternion beamspace, it is found that the quaternion transformation matrix offers us some additional degrees of freedom to handle the direction finding and polarization estimation. Using these degrees of freedom, we achieve the foregoing three goals as follows. First of all, we report two notable properties of a set of quaternion transformation matrices. One is to save the memory spaces for storing the data covariance matrix to $4 / 9$ of the traditional cases, and reduce the multiplications of the eigen-decomposition of the data covariance matrix from $O\left((6 M)^{3}\right)$ to $O\left((4 M)^{3}\right)$ for an array of $M$ vector-sensors.

Another is to blindly whiten the noise coherence of the selectric or magnetic components of each vector-sensor in an array which cannot be done by the methods in the literature.

After that, two types of the quaternion transformation matrices are analytically chosen to conduct two enhanced versions of the BB-MUSIC estimator. Such two estimators are capable of decoupling the estimations of polarization parameters from those of the direction angles. One of the proposed BB-MUSIC estimators, termed as DOA-BB-MUSIC, is able to estimate the direction angles of the sources without any polarization information of them. However, theoretical analyses also indicate that the DOA-BB-MUSIC estimator cannot estimate the polarization parameters either. Then another version of the enhanced BB-MUSIC estimator, nominated as DOA-PBB-MUSIC estimator, is presented to fulfill both the direction and polarization angle. The DOA-P-BB-MUSIC estimator requires a $2 \mathrm{D}$ search to find the direction angles first. After that the polarization parameters are obtained by another $2 \mathrm{D}$ search or finding the optimal solutions of the formulated linear equations. However, the abovementioned estimators can only naturally whiten the noise coherence of the electric or magnetic components of each vector-sensor in an array. To construct a biquaternion beamspace, which can be used to blindly whiten all of the noise coherence of the electric and magnetic components of each vector-sensor in an array, an optimal problem with a solution is given. Based on such a constructed biquaternion beamspace, an enhanced DOA-PBB-MUSIC estimator, nominated as DOA-PB-BB-MUSIC estimator, is then proposed for the direction findings and polarization estimations. The performances of the proposed estimators are tested by simulations, which confirm the correctness and effectiveness of our theoretical analyses.

Besides, the analytical results of the quaternion transformation matrix also show that the BQ-MUSIC in [13] can be considered as a special case of our BB-MUSIC estimator. Using the biquaternion beamspace theory presented herein, the reasons why the BQ-MUSIC estimator in [13] is robust to coherent noise and polarization errors are analytically presented by comparing the covariance of the complex and biquaternion algorithms.

The rest of the paper is organized as follows. In Section 2, the biquaternion beamspace and its measurement model are given by applying a quaternion transformation matrix to the traditional long vector model. Then in Section 3, the general form of our BB-MUSIC estimator is derived, the techniques to de-correlate the 
noise coherence are presented. In Section 4, two extended versions of BB-MUSIC estimator focusing on decoupling the polarization estimation from the direction finding are presented. The construction method of the biquaternion transformation matrix is introduced in Section 5. Simulation and comparison results with other estimators are reported in Section 6. Section 7 concludes this work.

\section{Biquaternion beamspace and its measurement model}

Similar to [13], we will use $\mathbb{R}, \mathbb{C}, \mathbb{H}$, and $\mathbb{H}_{\mathbb{C}}$ to denote the sets of real numbers, complex numbers, quaternions, and biquaternions, respectively.

\subsection{The long vector model}

Suppose that the completely polarized plane waves from $K$ narrow-band sources, traveling through a nonconductive homogenous isotropic medium, are impinging on an array of $M(M \geq K)$ identically oriented electromagnetic vector-sensors locating irregularly in a $3 \mathrm{D}$ region. Let the azimuth and elevation angles of the $k$ th source be $\phi_{k}$ and $\theta_{k}$, respectively. Thus, $\phi_{k} \in[0,2 \pi)$, and $\theta_{k} \in$ $\left[-\frac{\pi}{2}, \frac{\pi}{2}\right]$. The spatial phase factor for the $k$ th source to the $l$ th vector-sensor centered at location $\left(x_{l}, y_{l}, z_{l}\right)$ can be written as

$$
q_{l}\left(\theta_{k}, \phi_{k}\right)=e^{I \frac{2 \pi}{\lambda}\left(x_{l} \sin \theta_{k} \cos \phi_{k}+y_{l} \sin \theta_{k} \sin \phi_{k}+z_{l} \sin \phi_{k}\right)}
$$

where $\lambda$ is the length of the electromagnetic wave and $I$ is the imaginary unit of a complex number ${ }^{1}$.

The spatial response, in matrix notation, of the signal from the $k$ th source to the $l$ th vector-sensor can be expressed by [4-6]

$$
q_{l}\left(\theta_{k}, \phi_{k}\right) \underbrace{[\begin{array}{cc}
\left.\boldsymbol{g}_{k}, \gamma_{k}, \eta_{k}\right)= \\
\left.\begin{array}{cc}
-\sin \phi_{k} & \cos \phi_{k} \cos \theta_{k} \\
\cos \phi_{k} & \sin \phi_{k} \cos \theta_{k} \\
0 & -\sin \theta_{k} \\
-\cos \phi_{k} \cos \theta_{k} & -\sin \phi_{k} \\
-\sin \phi_{k} \cos \theta_{k} & \cos \phi_{k} \\
\sin \theta_{k} & 0
\end{array}\right]
\end{array} \underbrace{\left[\begin{array}{l}
\cos \gamma_{k} \\
\sin \gamma_{k} e^{I \eta_{k}}
\end{array}\right]}_{P\left(\gamma_{k}, \eta_{k}\right)}}_{V\left(\theta_{k}, \phi_{k}\right)}
$$

and $\eta \in[-\pi, \pi)$ are, respectively, the auxiliary polarization and phrase difference angles of the $k$ th source.

For the general case of $K$ narrow-band sources impinging on the array, the six-component vector measurements of $l$ th vector-sensor is given by [4]

$$
\boldsymbol{Y}_{l}(t)=\left[\begin{array}{l}
\boldsymbol{e}_{l}(t) \\
\boldsymbol{h}_{l}(t)
\end{array}\right]=\sum_{k=1}^{K} \boldsymbol{g}_{l}\left(\theta_{k}, \phi_{k}, \gamma_{k}, \eta_{k}\right) S_{k}(t)+\boldsymbol{N}_{l}(t)
$$

where $\boldsymbol{e}_{l}(t)=\left[e_{l x}(t) e_{l y}(t) e_{l z}(t)\right]^{T}$ and $\boldsymbol{h}_{l}(t)=\left[h_{l x}(t)\right.$ $\left.h_{l y}(t) h_{l z}(t)\right]^{T}\left((\cdot)^{T}\right.$ denoting the vector transpose) are, respectively, the three-component electric-field and magnetic-field measurement vectors of the $l$ th vectorsensor, $N_{l}$ is the additive complex noise, and $S_{k}(t)$ is the complex envelope (including amplitude and phase [4]) of the $k$ th source, i.e.,

$$
S_{k}(t)=\left|S_{k}(t)\right| e^{I \varphi_{k}}
$$

where $|\cdot|$ is the amplitude of a complex number and $\varphi_{k}$ is the $k$ th source's random carrier phase.

Let $\boldsymbol{Y}(t), \boldsymbol{S}(t)$, and $\boldsymbol{N}(t)$ be the column vectors describing the entire array's received signals, source incident signals, and noise, respectively, i.e.,

$$
\begin{gathered}
\boldsymbol{Y}(t)=\left[\begin{array}{llll}
\boldsymbol{Y}_{1}^{T}(t) & \boldsymbol{Y}_{2}^{T}(t) & \cdots & \boldsymbol{Y}_{M}^{T}(t)
\end{array}\right]^{T} \\
\boldsymbol{S}(t)=\left[\begin{array}{llll}
\boldsymbol{S}_{1}^{T}(t) \boldsymbol{S}_{2}^{T}(t) & \cdots & \boldsymbol{S}_{M}^{T}(t)
\end{array}\right]^{T} \\
\boldsymbol{N}(t)=\left[\begin{array}{llll}
\boldsymbol{N}_{1}^{T}(t) \boldsymbol{N}_{2}^{T}(t) & \cdots & \boldsymbol{N}_{M}^{T}(t)
\end{array}\right]^{T}
\end{gathered}
$$

The measurements of an electromagnetic vector-sensor array can thus be expressed as [4]

$$
\begin{aligned}
\boldsymbol{Y}(t) & =\sum_{k=1}^{K} \boldsymbol{q}\left(\theta_{k}, \phi_{k}\right) \otimes \boldsymbol{V}\left(\theta_{k}, \phi_{k}\right) \boldsymbol{P}\left(\gamma_{k}, \eta_{k}\right) S_{k}(t)+\boldsymbol{N}(t) \\
& =\boldsymbol{A} \boldsymbol{S}(t)+\boldsymbol{N}(t)
\end{aligned}
$$

where $\otimes$ is the Kronecker product and $A$ is the $6 M \times K$ matrix,

$$
\boldsymbol{A}=\left[\boldsymbol{a}_{1}, \boldsymbol{a}_{2}, \cdots, \boldsymbol{a}_{K}\right]
$$

with $\boldsymbol{a}_{k}=\boldsymbol{q}\left(\theta_{k}, \phi_{k}\right) \otimes \boldsymbol{V}\left(\theta_{k}, \phi_{k}\right) \boldsymbol{P}\left(\gamma_{k}, \eta_{k}\right)$,

$\boldsymbol{q}\left(\theta_{k}, \phi_{k}\right)=\left[q_{1}\left(\theta_{k}, \phi_{k}\right), q_{2}\left(\theta_{k}, \phi_{k}\right), \cdots, q_{M}\left(\theta_{k}, \phi_{k}\right)\right]^{T}$. The matrix $\boldsymbol{A}$ is generally assumed to be of full rank. It defines a $K$-dimensional signal subspace in a $6 M$ dimensional space [6].

From (5), it can be seen that $\boldsymbol{q}\left(\theta_{k}, \phi_{k}\right)$ depends on both the direction angles of the $k$ th source and the locations of the vector-sensors. It has an effect of spatial and temporal sampling of the $k$ th source's signals [3]. $V\left(\theta_{k}, \phi_{k}\right)$ depends only on the direction angles. It contains the source's direction information resulting from the spatial collocation of the vector-sensor's six constituent antennas. $\boldsymbol{P}\left(\theta_{k}, \phi_{k}\right)$ only relies on the polarization angles. It records the polarization information of the $k$ th source. Thus, it is explicit that an electromagnetic vector-sensor array records more source information than the scalarsensor one [2], because a scalar-sensor array only samples the source signals spatially and temporally [3].

When the source signals $S(t)$ and noise $N(t)$ are two independent stochastic processes, the spectral matrix 
$\boldsymbol{R}_{y}(t)$ of $(5)$ is given by $[2,5,6]$

$$
\begin{aligned}
\boldsymbol{R}_{y}(t) & =\mathbb{E}\left\{\boldsymbol{Y}(t) \boldsymbol{Y}^{H}(t)\right\} \\
& =\boldsymbol{A} \mathbb{E}\left\{\boldsymbol{S}(t) \boldsymbol{S}^{H}(t)\right\} A^{H}+\mathbb{E}\left\{\boldsymbol{N}(t) \boldsymbol{N}^{H}(t)\right\}
\end{aligned}
$$

where $\mathbb{E}\{\cdot\}$ is the mathematical expectation operator and $(\cdot)^{H}$ is the conjugate transpose of a complex matrix/vector.

Since the six measurements of each vector-sensor are concatenated into a $6 M \times 1$ long vector, (5) is also called as the long vector model (LV-MODEL) of an electromagnetic vector-sensor array in [11, 13]. As (5) shares the same form as the measurement model of a scalarsensor array [2, 3], the traditional MUSIC estimator for scalar-sensor array processing can directly applied to the vector-sensor case except for the fact that a $4 D$ search over the parameters $\left(\theta_{k}, \phi_{k}, \gamma_{k}, \eta_{k}\right)$ is needed. This vectorsensor version of the MUSIC estimator is often called as the long vector MUSIC (LV-MUSIC) estimator $[11,13]$.

\subsection{Biquaternion beamspace and its measurement model} For large arrays (i.e., a large $M$ ), the implementation of the signal subspace based algorithms require an $O\left((6 M)^{3}\right)$ eigendecomposition [10]. To achieve a significant reduction in computation time, an efficacious way is to project the original data into a lower dimension beamspace via a transformation matrix [8-10]. The parameter estimations are then carried out on the lower dimension beamspace data. The known transformation matrices, such as the spatio-polarizational [5] and standard Fourier [10] ones, are the complex ones. Unlike the traditional beamspace techniques, a quaternion transformation matrix is here used to project the original array data into our biquaternion beamspace. The measurement model in our biquaternion beamspace is defined as

$$
\boldsymbol{Z}(t)=\boldsymbol{T} \boldsymbol{Y}(t)=\boldsymbol{T A S}(t)+\boldsymbol{T N}(t)
$$

where $\boldsymbol{T} \in \mathbb{H}^{J \times 6 M}(K<J<6 M)$ is a quaternion matrix with orthogonal rows. That is $\boldsymbol{T} \boldsymbol{T}^{\triangleleft}=\boldsymbol{I}_{J}$, where $\triangleleft$ denotes the transpose-conjugate of a quaternion matrix as defined in [11] and $\boldsymbol{I}_{J}$ the $J \times J$ identity matrix. Meanwhile, the transformation matrix $\boldsymbol{T}$ in (8) plays the role as a beamformer to project the original array data $\boldsymbol{Y}(t)$ to our biquaternion beamspace. As $Y(t)$ is a complex vector in the LV-MODEL, $Z(t) \in \mathbb{H}_{c}^{J \times 1}$ is a biquaternion vector [13]. Correspondingly, the spectral matrix of the biquaternion beamspace output is given by

$$
\begin{aligned}
\boldsymbol{R}_{z}(t) & =\mathbb{E}\left\{Z(t) \boldsymbol{Z}^{\dagger}(t)\right\} \\
& =\boldsymbol{T} \boldsymbol{A} \mathbb{E}\left\{\boldsymbol{S}(t) \boldsymbol{S}^{H}(t)\right\} \boldsymbol{A}^{H} \boldsymbol{T}^{\triangleleft}+\boldsymbol{T} \mathbb{E}\left\{\boldsymbol{N}(t) \boldsymbol{N}^{H}(t)\right\} \boldsymbol{T}^{\triangleleft}
\end{aligned}
$$

where ${ }^{\dagger}$ is the transpose-conjugate of a biquaternion matrix/vector $[13,21]$.
Compare (7) with (9), one can see that the transformation matrix $\boldsymbol{T}$ in (9) offers us additional degrees of freedom to handle the spectral matrix $\boldsymbol{R}_{z}(t)$. In the next two sections, we aim at utilizing the additional freedom to achieve the three goals simultaneously, i.e., reducing the memory spaces and computation efforts, separating the sources' direction and polarization parameter searches, and improving the robustness of the MUSIC-like estimator to coherent corrupting noise.

\section{The biquaternion beamspace MUSIC estimator}

Before addressing how to determine the transformation matrix $\boldsymbol{T}$ in (9) for our three goals, let us first give the biquaternion beamspace MUSIC (BB-MUSIC) estimator.

\subsection{BB-MUSIC estimator}

Suppose that $\boldsymbol{T} \mathbb{E}\left\{\boldsymbol{N}(t) \boldsymbol{N}(t)^{H}\right\} \boldsymbol{T}^{\triangleleft}=\sigma^{2} \boldsymbol{I}_{J}$.

$\sigma^{2}$ is the observation noise power of the $l$ th vectorsensor.

In this way, (9) can be rewritten as

$$
\boldsymbol{R}_{z}(t)=\boldsymbol{T A} \mathbb{E}\left\{\boldsymbol{S}(t) \boldsymbol{S}^{H}(t)\right\} \boldsymbol{A}^{H} \boldsymbol{T}^{\triangleleft}+\sigma^{2} \boldsymbol{I}_{J}
$$

Similar to the scalar-sensor case, we need to constrain that $\mathbb{E}\left\{\boldsymbol{S}(t) \boldsymbol{S}^{H}(t)\right\}$ is a nonsingular matrix and $\boldsymbol{T A}$ is full rank in order to develop the MUSIC-like estimator. Using the biquaternion matrix eigen-decomposition (EVD) techniques as given in [13] or [20] $]^{2}$, the EVD of the Hermitian biquaternion matrix $\boldsymbol{R}_{z}(t)$ can be obtained as

$$
\boldsymbol{R}_{z}(t)=\boldsymbol{E}_{s} \boldsymbol{D}_{s} \boldsymbol{E}_{s}^{\dagger}+\boldsymbol{E}_{n} \boldsymbol{D}_{n} \boldsymbol{E}_{n}^{\dagger}
$$

where $\boldsymbol{E}_{s} \in \mathbb{H}_{\mathbb{C}}^{2 M \times 2 K}$ and $\boldsymbol{E}_{n} \in \mathbb{H}_{\mathbb{C}}^{2 M \times(4 M-2 K)}$ are, respectively, eigenvectors corresponding to the signal and noise subspaces, $\boldsymbol{D}_{s} \in \mathbb{R}^{2 K \times 2 K}$ and $\boldsymbol{D}_{n} \in \mathbb{R}^{(4 M-2 K) \times(4 M-2 K)}$ are two diagonal matrices whose diagonal elements are, respectively, the eigenvalues associated with $\boldsymbol{E}_{s}$ and $\boldsymbol{E}_{n}$. Projecting $\boldsymbol{T} \boldsymbol{a}_{k}$ on the noise subspace, we have ${ }^{3}$

$$
\left(\boldsymbol{T} \boldsymbol{a}_{k}\right)^{\dagger} \boldsymbol{E}_{n}=\mathbf{0}_{4 M-2 K},(k=1,2, \cdots K)
$$

where $\mathbf{0}_{4 M-2 K}$ is a $4 M-2 K$ dimensional row vector with all zero elements. The BB-MUSIC estimator consists of finding the set of parameters $\Omega_{k}=\left(\theta_{k}, \phi_{k}, \gamma_{k}, \eta_{k}\right)$ so that the following function is maximized:

$$
\mathcal{F}_{\mathrm{BB}}\left(\Omega_{k}\right)=\frac{1}{\left\|\left(\boldsymbol{T} \boldsymbol{a}_{k}\right)^{\dagger} \boldsymbol{E}_{n}\right\|^{2}}=\frac{1}{\mathbb{S}\left\{\left(\boldsymbol{T} \boldsymbol{a}_{k}\right)^{\dagger} \boldsymbol{E}_{n} \boldsymbol{E}_{n}^{\dagger}\left(\boldsymbol{T} \boldsymbol{a}_{k}\right)\right\}}
$$

where $\|\cdot\|$ denotes the norm of a biquaternion vector as given in [13] and $\mathbb{S}\{\cdot\}$ denotes the operator taking the scalar part of a biquaternion matrix. Let $\boldsymbol{\Omega}\left(\theta_{k}, \phi_{k}\right)=$ $\boldsymbol{T} \boldsymbol{q}\left(\theta_{k}, \phi_{k}\right) \otimes \boldsymbol{V}\left(\theta_{k}, \phi_{k}\right),(13)$ can be rewritten as

$$
\mathcal{F}_{\mathrm{BB}}\left(\Omega_{k}\right)=\frac{1}{\boldsymbol{P}^{H}\left(\gamma_{k}, \eta_{k}\right) \boldsymbol{\Xi}\left(\theta_{k}, \phi_{k}\right) \boldsymbol{P}\left(\gamma_{k}, \eta_{k}\right)}
$$


where

$$
\boldsymbol{\Xi}\left(\theta_{k}, \phi_{k}\right)=\mathbb{S}\left\{\left[\boldsymbol{\Theta}\left(\theta_{k}, \phi_{k}\right)\right]^{\dagger} \boldsymbol{E}_{n} \boldsymbol{E}_{n}^{\dagger}\left[\boldsymbol{\Theta}\left(\theta_{k}, \phi_{k}\right)\right]\right\}
$$

$\boldsymbol{P}\left(\gamma_{k}, \eta_{k}\right)$ can refer to (2). It can be seen that (15) depends only on the direction angular parameters of the sources.

It is understood that the prerequisite of the traditional subspace estimation algorithms, such as MUSIC $[2,3]$, and ESPRIT [6, 23], is that the additive noise $N(t) \in$ $\mathbb{C}^{6 M \times 1}$ in (7) is a complex Gaussian distributed random vector with a covariance matrix

$$
\mathbb{E}\left\{\boldsymbol{N}(t) \boldsymbol{N}^{H}(t)\right\}=\sigma^{2} \boldsymbol{I}_{6 M}
$$

It means that their noise components are required to be noncoherent with one another. For the BB-MUSIC estimator, such a prerequisite becomes as follows:

$$
\boldsymbol{T} \mathbb{E}\left\{\boldsymbol{N}(t) \boldsymbol{N}^{H}(t)\right\} \boldsymbol{T}^{\triangleleft}=\sigma^{2} \boldsymbol{I}_{2 M}
$$

which is the basic assumption of our BB-MUSIC estimator as given at the very beginning of this subsection. Moreover, if (17) has to be satisfied while (16) holds, one should, obviously, let $\boldsymbol{T} \boldsymbol{T}^{\triangleleft}=\boldsymbol{I}_{2 M}$. Namely, the rows of $\boldsymbol{T}$ are orthogonal to each other. This is also the reason why our biquaternion beamspace (8) requires $\boldsymbol{T} \boldsymbol{T}^{\triangleleft}=\boldsymbol{I}_{2 M}$. In the next subsection, we will show that, if the transformation matrix $\boldsymbol{T}$ is chosen or constructed properly, (17) may still hold even if $\mathbb{E}\left\{\boldsymbol{N}(t) \boldsymbol{N}^{H}(t)\right\}$ in (16) is not a diagonal matrix. It implies that the developed BB-MUSIC may be applied to some coherent corrupting noise cases.

\subsection{Robustness to coherent noise components}

Before rendering our analyses, let us first introduce a mapping. Define $\boldsymbol{t}=\left[\begin{array}{ll}i & j\end{array}\right]$, where $i, j$ and $k$ denote three quaternion imaginary units respectively. Thus $t$ is a quaternion vector. Let a $3 \times 3 \mathrm{real} /$ complex matrix $\boldsymbol{R}$ given by

$$
\boldsymbol{R}=\left[\begin{array}{l}
r\langle 1,1\rangle r\langle 1,2\rangle r\langle 1,3\rangle \\
r\langle 2,1\rangle r\langle 2,2\rangle r\langle 2,3\rangle \\
r\langle 3,1\rangle r\langle 3,2\rangle r\langle 3,3\rangle
\end{array}\right]
$$

By the operation rules of the biquaternion algebra, we can define the mapping as follows:

$$
\begin{aligned}
\boldsymbol{R}_{b}= & \boldsymbol{t} \boldsymbol{R} \boldsymbol{t}^{\triangleleft} \\
= & (r\langle 1,1\rangle+r\langle 2,2\rangle+r\langle 3,3\rangle) \\
& +(r\langle 2,3\rangle-r\langle 3,2\rangle) i+(r\langle 3,1\rangle-r\langle 1,3\rangle) j \\
& +(r\langle 1,2\rangle-r\langle 2,1\rangle) k
\end{aligned}
$$

The mapping of (19) transforms a $3 \times 3$ real or complex matrix $\boldsymbol{R}$ into a quaternion or biquaternion number $\boldsymbol{R}_{b}$. When $\boldsymbol{R}$ is a symmetric matrix, i.e., $\boldsymbol{R}=\boldsymbol{R}^{T}, \boldsymbol{R}_{b}$ is a real number equaling to $(r\langle 1,1\rangle+r\langle 2,2\rangle+r\langle 3,3\rangle)$. When $\boldsymbol{R}$ is a general complex or real matrix, $\boldsymbol{R}_{b}$ is a biquaternion or quaternion number. Moreover, if $\boldsymbol{R}$ is the covariance matrix of a three dimensional random noise vector, $\boldsymbol{R}$ will be mapped into a real number equaling to the sum of the three variance items of $\boldsymbol{R}$, while the covariance items are canceled out by (19). From this point, the mapping in (19) can naturally whiten the coherence among a three-component random noise vector.

Rewrite the noise covariance matrix $\boldsymbol{R}_{n}=$ $\mathbb{E}\left\{\boldsymbol{N}(t) \boldsymbol{N}^{H}(t)\right\} \in \mathbb{R}^{6 M \times 6 M}$ in (7) as the following blockwise partition form

$$
\boldsymbol{R}_{n}=\left[\begin{array}{ccc}
\boldsymbol{R}_{n}(1,1) & \cdots & \boldsymbol{R}_{n}(1,2 M) \\
\vdots & & \vdots \\
\boldsymbol{R}_{n}(2 M, 1) & \cdots & \boldsymbol{R}_{n}(2 M, 2 M)
\end{array}\right]
$$

where each block entry is a $3 \times 3$ matrix. $\boldsymbol{R}_{n}(2 l-1,2 l-1)$ and $\boldsymbol{R}_{n}(2 l, 2 l)(l=1, \cdots, M)$ are, respectively, the noise covariance matrices (symmetrical matrices) of the electric-field antennas (short dipoles $[1,6]$ ) and the magnetic-field antennas (small loops $[1,6]$ ) of the $l$ th vector-sensor. $\boldsymbol{R}_{n}(m, n)(m \neq n)$ is the noise covariance matrix of the short dipoles and small loops of a vectorsensor or different vector-sensors.

Let $\boldsymbol{T}$ be a $2 M \times 6 M$ quaternion matrix with the following form

$$
\boldsymbol{T}=\frac{1}{\sqrt{3}} \boldsymbol{I}_{M} \otimes\left[\begin{array}{ll}
\boldsymbol{t} & 0 \\
0 & \boldsymbol{t}
\end{array}\right]
$$

From (9), it is known that the noise covariance matrix $\boldsymbol{R}_{n}$ of the biquaternion beamspace can be rewritten as

$$
\begin{aligned}
\boldsymbol{R}_{b n} & =\boldsymbol{T} \boldsymbol{R}_{n} \boldsymbol{T} \\
& =\left[\begin{array}{ccc}
\boldsymbol{R}_{b n}(1,1) & \cdots & \boldsymbol{R}_{b n}(1,2 M) \\
\vdots & & \vdots \\
\boldsymbol{R}_{b n}(2 M, 1) & \cdots & \boldsymbol{R}_{b n}(2 M, 2 M)
\end{array}\right] \in \mathbb{H}^{2 M \times 2 M}
\end{aligned}
$$

where

$$
\boldsymbol{R}_{b n}\langle m, n\rangle=\frac{1}{3} \boldsymbol{t} \boldsymbol{R}_{n}\langle m, n\rangle \boldsymbol{t}^{\triangleleft} \in \mathbb{H}
$$

is a quaternion number. Based on (19), one can conclude that

1. The noise variance sums of three short dipoles or small loops are represented by $\boldsymbol{R}_{b n}\langle m, n\rangle=\frac{1}{3} \boldsymbol{t} \boldsymbol{R}_{n}\langle m, n\rangle \boldsymbol{t}^{\triangleleft} \in \mathbb{R}$, in which their covariance items are canceled out.

2. $\boldsymbol{R}_{b n}\langle m, n\rangle=\frac{1}{3} \boldsymbol{t} \boldsymbol{R}_{n}\langle m, n\rangle \boldsymbol{t}^{\triangleleft} \in \mathbb{H}_{\mathbb{C}}(m \neq n)$ describes the noise coherence among the short dipoles and small loops of the same vector-sensor or that among the different vector-sensors. The three quaternion imaginary parts of (19) carry out three subtraction operations for the covariance items (positive real numbers) of $\boldsymbol{R}_{n}\langle m, n\rangle$. These operations can weaken the noise coherence because the difference of two positive numbers is smaller than either of them. 
Thus, when the transformation matrix is chosen as (21), the noise coherence among the electric-field or magnetic-field components of a vector-sensor can be totally whitened, while the noise coherence among other components is weaken (or partially decorrelated). As a result, our BB-MUSIC estimator can be extended to the coherent noise cases satisfying the following assumptions:

A1. the noise components of the different vector-sensors in an array are noncoherent one another.

A2. a vector-sensor's electric-field noise is noncoherent with its magnetic-field noise.

A3. the electric-field noise of a vector-sensor is allowed be coherent or noncoherent, and so is the magnetic-field noise.

With the above analytical results, it can be seen that the BB-MUSIC estimator will outperform the traditional long vector type estimators under the condition of coherent corrupting noise.

It should be mentioned that there are many different transformation matrices yielding the equivalent results as (19). In order to explain such a point, let us change the transformation matrix in (21) into the following forms as

$$
\boldsymbol{T}=\frac{1}{\sqrt{3}} \boldsymbol{I}_{M} \otimes\left[\begin{array}{cc}
\boldsymbol{t}_{a} & 0 \\
0 & \boldsymbol{t}_{b}
\end{array}\right]
$$

in which $\boldsymbol{t}_{a}$ and $\boldsymbol{t}_{b}$ are $1 \times 3$ quaternion vectors, whose three entries are unit quaternions orthogonal to each other, such as $\boldsymbol{t}_{a}=1 / \sqrt{3}[i j k]$ and $\boldsymbol{t}_{b}=1 / \sqrt{3}[k j i]$ or $\boldsymbol{t}_{a}=1 / \sqrt{3}[i j k]$ and $\boldsymbol{t}_{b}=\sqrt{2} / 6[\sqrt{3}(i+j) \sqrt{2}(i-$ $j+k)(i-j+k)]$. If one takes $\boldsymbol{t}_{a}=1 / \sqrt{3}[i j k]$ and $\boldsymbol{t}_{b}=1 / \sqrt{3}\left[\begin{array}{lll}0 & 0 & 0\end{array}\right]$, it can be seen that the BQ-MUSIC is a special case of our biquaternion beamspace model by taking the transformation matrix as (24). In a general way, let $\boldsymbol{t}_{a}=\left[e_{1} e_{2} e_{3}\right], \boldsymbol{t}_{b}=\left[e_{4} e_{5} e_{6}\right]$, where $e_{1}, e_{2}, e_{3}$ and $e_{4}, e_{5}, e_{6}$ are any two unit quaternion orthogonal vectors, respectively. Thus, $\boldsymbol{t}_{a}$ and $\boldsymbol{t}_{b}$ can be changed into more general

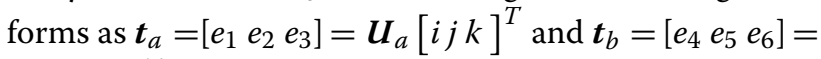
$\boldsymbol{U}_{b}[i j k]^{T}$, where $\boldsymbol{U}_{a}$ and $\boldsymbol{U}_{b}$ are any $3 \times 3$ real orthogonal matrix. By directly calculation, one can easily verify that (19) is still hold for these chosen $\boldsymbol{t}_{a}$ and $\boldsymbol{t}_{b}$. The differences are that the last three terms in (19) will be changed with the different $\boldsymbol{T}$ in (24). Even so, our BB-MUSIC estimator can still work correctly under the assumptions A1, $\mathrm{A} 2$ and $\mathrm{A} 3$.

As a side note, it should be pointed out the fact that the mapping of (19) can also reduce the memory spaces of $\boldsymbol{R}$ in (18). Obviously, $\boldsymbol{R}$ needs 9 or 18 memory units to store a $3 \times 3$ real or complex matrix, whereas 4 or 8 memory units are enough for storing $\boldsymbol{R}_{b}$. As a result, when taking the entire array into consideration, (9) indicates that $32 M^{2}$ memory units are required to restore, while $72 M^{2}$ memory units are required to restore $\boldsymbol{R}_{y}(t)$.
The point is not new because Miron et al. in [13] have given such results by the calculation rules of biquaternions. Furthermore, it should be noticed that our biquaternion beamspace also helps to reduce the computation complexity of the EVD for the MUSIC like estimators. It has been understood that the EVD of a $M \times M$ complexmatrix requires $O\left(M^{3}\right)$ multiplications [10]. It implies that the multiplications for the EVD of $\boldsymbol{R}_{y}(t)$ are $O\left((6 M)^{3}\right)$. By contrast, the multiplications for the EVD of $\boldsymbol{R}_{z}(t)$ are $O\left((4 M)^{3}\right)$. This is because the EVD of the $2 M \times 2 M$ biquaternion matrix are obtained via the EVD of its complex representation matrix with a size $4 M \times 4 M[20]$.

\section{Direction finding and polarization estimation in biquaternion beamspace}

In this Section, two distinct versions of the BB-MUSIC estimator based on the different transformation matrix in (24) are reported. One is developed to estimate the source direction angles without any prior polarization information. Another is built to use a 2D search to find the source direction angles first and then polarization ones.

\subsection{Decoupling polarization angles from direction finding} Unlike the self-initiating MUSIC estimator in [5] decoupling the polarization estimation from direction finding via the spatio-polarizational beamforming, we approach to that by the transformation matrix as given in (24).

If the transformation matrix in (24) satisfies $\boldsymbol{t}_{a}=\boldsymbol{t}_{b}=$ $\left[\begin{array}{lll}e_{1} & e_{2} & e_{3}\end{array}\right]$, we have

$$
\boldsymbol{T}=\frac{1}{\sqrt{3}} \boldsymbol{I}_{M} \otimes\left[\begin{array}{cccccc}
e_{1} & e_{2} & e_{3} & 0 & 0 & 0 \\
0 & 0 & 0 & e_{1} & e_{2} & e_{3}
\end{array}\right]
$$

Thus, we have the following theorem for decoupling the polarization estimation from the direction finding:

Theorem 1 When $\gamma \neq \pi / 4$ and $\eta \neq \pm \pi / 2,\left(\boldsymbol{T a}_{k}\right)^{\dagger} \boldsymbol{E}_{n}=$ $\mathbf{0}_{4 M-2 K}$ is equivalent to

$$
\left(\boldsymbol{q}\left(\theta_{k}, \phi_{k}\right) \otimes\left[\begin{array}{l}
1 \\
u_{k}
\end{array}\right]\right)^{\dagger} \boldsymbol{E}_{n}=\mathbf{0}_{4 M-2 K},(k=1,2, \cdots K)
$$

where $u_{k}=\cos \phi_{k} \cos \theta_{k} i+\sin \phi_{k} \cos \theta_{k} j \quad+$ $\sin \theta_{k} k,(k=1,2, \ldots, K)$

Proof See Appendix 1.

From Theorem 1, it can be seen that (26) is nothing to do with the polarization parameters $\left(\gamma_{k}, \eta_{k}\right)$ because both $\boldsymbol{q}\left(\theta_{k}, \phi_{k}\right)$ and $u_{k}$ only depend on the direction angular 
parameters $\left(\theta_{k}, \phi_{k}\right)$ of the sources. Thus, the expression,

$$
\mathcal{F}_{\mathrm{DOA}-\mathrm{BB}}\left(\theta_{k}, \phi_{k}\right)=\frac{1}{\left\|\left(\boldsymbol{q}\left(\theta_{k}, \phi_{k}\right) \otimes\left[\begin{array}{l}
1 \\
u_{k}
\end{array}\right]\right)^{\dagger} \boldsymbol{E}_{n}\right\|^{2}}
$$

can first be used to find the directions angles of the sources with polarization parameters $\gamma_{k} \neq \pi / 4$ and $\eta_{k} \neq$ $\pm \pi / 2$. After that, one substitutes $\gamma_{k}=\pi / 4$ and $\eta_{k}=$ $\pm \pi / 2$ into (14) and then use it to search the directions angles of the sources with the two polarization states. In this way, the traditional 4D search in the MUSIC methods is reduced to three 2D searches for our estimators (27) and (14). As (27) can only find the direction of arrival (DOA) of the sources, we call it as the DOA-BB-MUSIC estimator for the latter discussion convenience.

Although the transformation matrix of (25) enables us to decouple the polarization parameters from the source direction finding, the following Theorem 2 tells us that the estimates of the polarization parameters cannot be handled via the transform matrix as (25).

Theorem 2 If the transformation matrix of the biquaternion beamspace is taken as (25), $\Xi\left(\theta_{k}, \phi_{k}\right)$ in (15) will be changed into

$$
\mathbf{\Xi}\left(\theta_{k}, \phi_{k}\right)=\left[\begin{array}{cc}
a & -b I \\
b I & a
\end{array}\right]
$$

where $a$ and $b$ are two real numbers depending only on $\left(\theta_{k}, \phi_{k}\right)$. Moreover, the larger $4 M-2 K$, the smaller $|b / a|$. Inserting (28) into (14), the BB-MUSIC spectrum can be rewritten as

$$
\mathcal{F}_{B B}\left(\Omega_{k}\right)=\frac{1}{a\left(1+\frac{b}{a} \sin 2 \gamma_{k} \sin \eta_{k}\right)}
$$

Proof See Appendix 2.

It can be seen that, although the shape of the spectrum defined by $\mathcal{F}_{\mathrm{BB}}\left(\Omega_{k}\right)$ changes with $a, b$, and $\sin 2 \gamma_{k} \sin \eta_{k}$, the extrema of (29) for polarization parameters are only taken at fix locations with $\left|\sin 2 \gamma_{k} \sin \eta_{k}\right|=1$, i.e., $(\gamma=\pi / 4, \eta= \pm \pi / 2)$. It indicates that (29) cannot be used to find the polarization parameters when the transformation matrix is taken as (25).

As a matter of fact, the key point why (25) is incapable of estimating the polarization parameters is the form of $\boldsymbol{\Xi}\left(\theta_{k}, \phi_{k}\right)$ in (28) making the extrema of (29) fall away them. To develop an estimator capable of doing the direction finding and polarization estimation both, the transformation matrix different from (25) should be chosen to break the form of $\boldsymbol{\Xi}\left(\theta_{k}, \phi_{k}\right)$ in (28). We will investigate this topic in the next subsection.

\subsection{Direction finding and polarization estimation}

To maintain the polarization information in the BBMUSIC spectrum, let us consider the transformation matrices in (24) satisfying $\boldsymbol{t}_{a} \neq \boldsymbol{t}_{b}$ and introduce a new quaternion beamspace matrix $\boldsymbol{T}_{p}$ :

$$
\boldsymbol{T}_{p}=\frac{1}{\sqrt{3}} \boldsymbol{I}_{M} \otimes\left[\begin{array}{cccccc}
e_{1} & e_{2} & e_{3} & 0 & 0 & 0 \\
0 & 0 & 0 & e_{4} & e_{5} & e_{6}
\end{array}\right]
$$

One can very easily verify that $\boldsymbol{T}_{p}$ is a quaternion matrix with orthogonal rows and can make (19) hold. Using (30), the BB-MUSIC spectrum in (14) is changed into

$$
\begin{gathered}
\mathcal{F}\left(\Omega_{k}\right)=1 /\left(a_{\Lambda 1} \cos \gamma_{k}^{2}+a_{\Lambda 2} \sin \gamma_{k}^{2}+a_{\Lambda 12} \sin 2 \gamma_{k} \cos \eta_{k}\right. \\
\left.-b_{\Lambda 12} \sin 2 \gamma_{k} \sin \eta_{k}\right)
\end{gathered}
$$

Proof Reduction details:

\section{See Appendix 3.}

One can easy find from (31) that the polarization parameters cannot be decoupled. However, a 4D search can help for the direction finding and polarization angle estimations. In other words, the polarization parameters estimation is achievable via introducing the new biquaternion beamspace matrix $\boldsymbol{T}_{p}$. As the $4 \mathrm{D}$ search is time consuming and computation prohibitive, we next discuss how to decouple the polarization estimations of (31) from its direction finding.

Equation (12) can be rewritten as

$$
\left(\boldsymbol{T}_{p} \boldsymbol{a}_{k}\right)^{\dagger} \boldsymbol{E}_{n}=\mathbf{0}_{4 M-2 K},(k=1,2, \cdots K)
$$

and then (32) can be changed into

$$
\mathcal{F}\left(\Omega_{k}\right)=\frac{1}{\boldsymbol{P}^{H}\left(\gamma_{k}, \eta_{k}\right) \mathbf{\Xi}_{p}\left(\theta_{k}, \phi_{k}\right) \boldsymbol{P}\left(\gamma_{k}, \eta_{k}\right)}
$$

where $\boldsymbol{\Xi}_{p}\left(\theta_{k}, \phi_{k}\right)=\mathbb{S}\left\{\left[\boldsymbol{\Theta}\left(\theta_{k}, \phi_{k}\right)\right]^{\dagger} \boldsymbol{E}_{n} \boldsymbol{E}_{n}^{\dagger}\left[\boldsymbol{\Theta}\left(\theta_{k}, \phi_{k}\right)\right]\right\}$.

To maximize (33) over $\Omega_{k}$, we can first fix the direction $\left(\theta_{k}, \phi_{k}\right)$ and maximize (33) over polarization angles $\left(\gamma_{k}, \eta_{k}\right)$. In this way, the $4 \mathrm{D}$ search over $\Omega_{k}$ is reduced to a 2D search over $\left(\theta_{k}, \phi_{k}\right)$. This idea was first used by Ferrara and Parks in [24] for direction finding via diversely polarized antennas. Thus, the polarization decoupled spectrum of (33) is

$$
\mathcal{F}\left(\theta_{k}, \phi_{k}\right)=\left\{\max _{\gamma_{k}, \eta_{k}} \frac{1}{\boldsymbol{P}^{H}\left(\gamma_{k}, \eta_{k}\right) \boldsymbol{\Xi}_{p}\left(\theta_{k}, \phi_{k}\right) \boldsymbol{P}\left(\gamma_{k}, \eta_{k}\right)}\right\}
$$

Since $\boldsymbol{P}^{H}\left(\gamma_{k}, \eta_{k}\right) \boldsymbol{P}\left(\gamma_{k}, \eta_{k}\right)=1$ and $\boldsymbol{\Xi}\left(\theta_{k}, \phi_{k}\right)$ is a complex Hermitian matrix, according to the Rayleigh's principle [25], we obtain

$$
\max _{\gamma_{k}, \eta_{k}} \frac{1}{\boldsymbol{P}^{H}\left(\gamma_{k}, \eta_{k}\right) \boldsymbol{\Xi}_{p}\left(\theta_{k}, \phi_{k}\right) \boldsymbol{P}\left(\gamma_{k}, \eta_{k}\right)}=\frac{1}{\lambda_{\min }\left\{\mathbf{\Xi}_{p}\left(\theta_{k}, \phi_{k}\right)\right\}}
$$


where $\lambda_{\min }\left\{\mathbf{\Xi}_{p}\left(\theta_{k}, \phi_{k}\right)\right\}$ denotes the operator taking the smallest eigenvalue of the matrix $\boldsymbol{\Xi}_{p}\left(\theta_{k}, \phi_{k}\right)$. From (35), it can be seen that our the BB-MUSIC spectrum for DOA estimation is

$$
\mathcal{F}_{\mathrm{DOA}}\left(\theta_{k}, \phi_{k}\right)=\frac{1}{\lambda_{\min }\left\{\mathbf{\Xi}_{p}\left(\theta_{k}, \phi_{k}\right)\right\}}
$$

Once the direction angular $\left(\theta_{k}, \phi_{k}\right)$ is found by the 2D search of (36), one can further substitute $\left(\theta_{k}, \phi_{k}\right)$ into (33), perform a $2 \mathrm{D}$ search to find the polarization parameters belonging to the sources with direction parameters $\left(\theta_{k}, \phi_{k}\right)$.

Of course, when the DOA has been obtained, one can do polarization estimation with the LV-MUSIC. However, while $72 M^{2}$ memory units are required to restore $\boldsymbol{R}_{y}(t)$ in (7), it also increase the computation efforts of the eigendecomposition of the data covariance matrix.

In summary, when choosing the transformation matrix in (24) with $\boldsymbol{t}_{a} \neq \boldsymbol{t}_{b}$, we can first perform a 2D search of (36) to estimate the direction angles of the sources, then estimate the corresponding polarization parameters. To avoid confusion, we nominate this method as DOA and polarization estimation BB-MUSIC (DOA-PBB-MUSIC) estimator. Correspondingly, the techniques used in (36) can also be applied to the traditional LVMUSIC estimator. We will terms this new version LVMUSIC estimator as DOA-LV-MUSIC because it can be used to estimate the DOA of the sources without any polarization information.

\section{Construction of biquaternion beamspace matrix}

In Section 3, we have known that there are an infinite number of biquaternion beamspace matrices making (19) satisfied. When choosing the transformation matrix $\boldsymbol{T}$ as (25), the noise coherence among the electric field components or magnetic field ones can be eliminated. However, the noise coherence between the electric-field and magnetic-field components of each vector-sensor cannot be whitened. Thus, it is desirable to find an appropriate transformation matrix $\boldsymbol{T}_{p}$ as (30) which can whiten all of the coherent noise in each vector-sensor.

Assuming that the eigenvalues of the signal and noise in (11) are, respectively, $\lambda_{1}, \lambda_{2}, \cdots \lambda_{2 K}$ and $\lambda_{2 K+1}, \lambda_{2 K+2}, \cdots \lambda_{4 M}$ with $\lambda_{1} \geqslant \lambda_{2} \geqslant \cdots \geqslant \lambda_{4 M}$, it is well known that, if the noise in a vector array is a additive white noise, the eigenvalues of the noise subspace of an ideal data covariance matrix are equal, i.e. $\lambda_{2 K+1}=\lambda_{2 K+2}=\cdots=\lambda_{4 M}$. However, an ideal data covariance matrix with the infinite data for an application is unpractical. If the additional degrees of freedom offered to us by the quaternion transformation matrix can be employed to make the eigenvalues of the noise subspace as equal as possible, all of the coherent noise of vector-sensor reduced may be expected. Mathematically, it can be formulated as the following optimization problem

$$
\begin{array}{cc}
\min & \operatorname{var}\left(\lambda_{2 K+1}, \lambda_{2 K+2}, \cdots \lambda_{4 M}\right) \\
\text { s.t. } & \boldsymbol{T}_{p} \boldsymbol{T}_{p}{ }^{\triangleleft}=\boldsymbol{I}_{2 M}
\end{array}
$$

where $\operatorname{var}(\cdot)$ means to get the variance of the $\lambda_{2 K+1}, \lambda_{2 K+2}, \cdots \lambda_{4 M}$. The idea of (37) is that we try to find a biquaternion transformation matrix $\boldsymbol{T}_{p}$ which can make the noise eigenvalue changes of the transformed covariance matrix be as small as possible. It must be emphasized that the derivation of matrix $\boldsymbol{T}_{p}$ does not use any prior knowledge of noise. Nevertheless, extra computation cost must be paid.

Using the fmincon function, taking the active-set algorithm, in Matlab optimization toolbox, (37) can be solved. Once the biquaternion beamspace matrix $\boldsymbol{T}_{p}$ is got, the DOA parameters and polarization estimations can be obtained by using the same method as DOA-PBB-MUSIC algorithm. These procedures are named as DOA-PB-BB-MUSIC algorithm. Refer to (3.4) in [4], the Cramer-Rao bound (CRB) of the DOA-PB-BB-MUSIC algorithm in the presence of the coherent corrupting noise is given as

$\operatorname{CRB}(\Omega)=\frac{\sigma^{2}}{2 L}\left\{\operatorname{Re}\left[\operatorname{btr}\left(\left(\mathbf{1} \otimes \boldsymbol{U}_{b q}\right) \odot\left(\boldsymbol{D}_{b q}^{\dagger} \boldsymbol{\Pi}_{c b q} \boldsymbol{D}_{b q}\right)^{b T}\right)\right]\right\}^{-1}$

Proof See Appendix 4.

\section{Simulation results}

The performances of the three newly proposed estimators are evaluated in this section. Since both DOA-BBMUSIC and DOA-P-BB-MUSIC estimators are capable of decoupling the polarization estimation from the direction finding, their direction finding performances will be compared with two other polarization decoupled estimators, namely the DOA-LV-MUSIC and ESPRIT [23]. Besides, the polarization estimation performances of DOA-P-BB-MUSIC will also be compared with the ESPRIT estimator in [23].

In all the following simulations, a linear uniform array with all vector-sensors along the $x$-axis with 0.5 wavelength inter-element spacing is chosen.

To evaluate the performances of direction findings and polarization estimations, we define the composite root-mean-square (RMS) errors of the direction and polarization angles for the $K$ sources as $\left\{\left[\sum_{k=1}^{K}\left(\Delta \theta_{k}^{2}+\Delta \phi_{k}^{2}\right)\right] /(2 K)\right\}^{\frac{1}{2}}$ and $\left\{\left[\sum_{k=1}^{K}\left(\Delta \gamma_{k}^{2}+\Delta \eta_{k}^{2}\right)\right] /(2 K)\right\}^{\frac{1}{2}}$, 
respectively, where $\Delta \theta_{k}, \Delta \phi_{k}, \Delta \gamma_{k}$ and $\Delta \eta_{k}$ are, respectively, the estimation errors of $\theta_{k}, \phi_{k}, \gamma_{k}$ and $\eta_{k}$.

First, we test the direction finding performances of the proposed estimators. We consider an array of 15 vector-sensors and 4 uncorrelated narrow-band incident sources ${ }^{4}$ with the following simulation parameters:

$$
\begin{aligned}
& \phi_{1}=4.5 \mathrm{rad}, \theta_{1}=-0.8 \mathrm{rad}, \gamma_{1}=0.7 \mathrm{rad}, \eta_{1}=0.3 \mathrm{rad} \\
& \phi_{2}=3 \mathrm{rad}, \theta_{2}=-0.1 \mathrm{rad}, \gamma_{2}=0.1 \mathrm{rad}, \eta_{2}=-1.5 \mathrm{rad} \\
& \phi_{3}=3.8 \mathrm{rad}, \theta_{3}=0.4 \mathrm{rad}, \gamma_{3}=\pi / 4 \mathrm{rad}, \eta_{3}=\pi / 2 \mathrm{rad} \\
& \phi_{4}=1.5 \mathrm{rad}, \theta_{4}=0.7 \mathrm{rad}, \gamma_{4}=\pi / 4 \mathrm{rad}, \eta_{4}=-\pi / 2 \mathrm{rad}
\end{aligned}
$$

Noncoherent noise is injected with an $S N R=10 \mathrm{~dB}$, and 1000 snapshots are used. Among the four sources, the polarization angles of the first and second ones satisfy the requirements of Theorem 1 , while those of the third and fourth sources do not satisfy the ones. Since the LV-MUSIC estimator requires to perform a 4D search, we just fix its polarization parameters to $\gamma_{4}$ and $\eta_{4}$ to perform a $2 \mathrm{D}$ search, while the other estimators perform $2 \mathrm{D}$ searches directly because they are capable of decoupling the polarization estimations from the direction finding. From Fig. 1a, it can be seen that the LV-MUSIC estimator can only correctly find the fourth sources, because we fix the polarization parameters to the fourth sources and perform a $2 \mathrm{D}$ search. In Fig. $1 \mathrm{~b}$ and c, one can find that both the DOA-LV-MUSIC and DOA-P-BB-MUSIC successfully find the fourth sources via $2 \mathrm{D}$ searches over the direction angles. For the DOA-BB-MUSIC estimator, we first employ a 2D search with (27) to find the direction angles of the first and second source because their polarization angles satisfy the requirements of Theorem 1. Its estimate results are shown in Fig. 1d. Then, as depicted in Fig. 1e and f, respectively, we use another two $2 \mathrm{D}$ searches to estimate the direction angles of the third and fourth sources by inserting $\gamma=\pi / 4$ and $\eta= \pm \pi / 2$ into (14). As it can be seen in Fig. 1e, except for the highest peak generated by the third source, two lower peaks of the first and second sources also emerge. Similar phenomenon also accompanies with the fourth source as given in Fig. 1f. This is due to the fact that (29) depends slightly on $\left(\gamma_{k}, \eta_{k}\right)$ when $|b / a|$ is small. Moreover, the smaller $|b / a|$, the higher possibility as we can see the first and second sources in Fig. 1e and f. If all sources' directions and polarization angles are different from each other ${ }^{5}$, this phenomenon will not induce any ambiguities by simply adding the spectrums of Fig. $1 \mathrm{~d}-\mathrm{f}$ together, as shown in Fig. 1g. Using the QQ-MUSIC estimator, the peak of the fourth source is lower than the others, as shown in Fig. 1h. The above results confirm the analytical correctness of our DOA-BB-MUSIC and DOA-P-BB-MUSIC estimators.

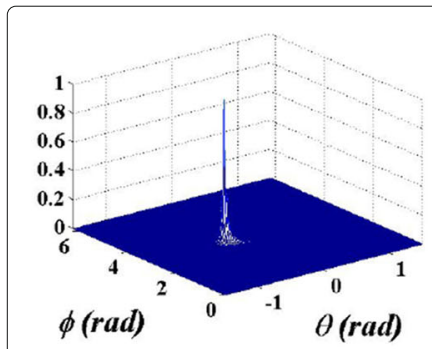

a

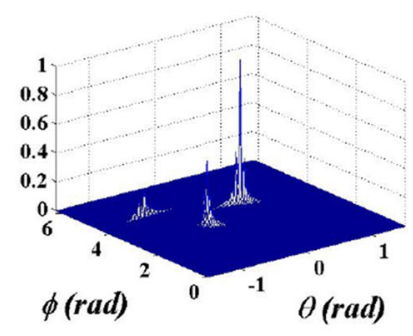

e

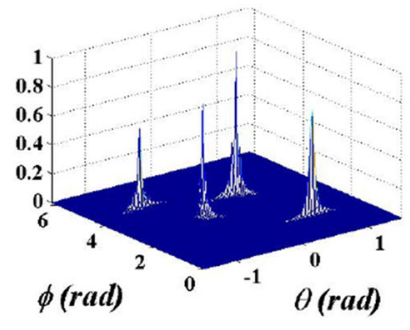

b

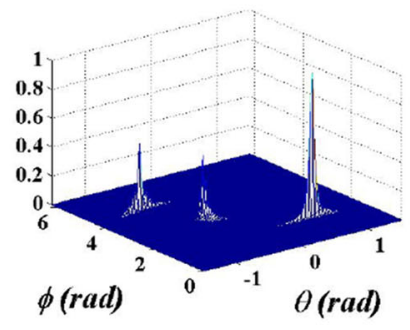

f

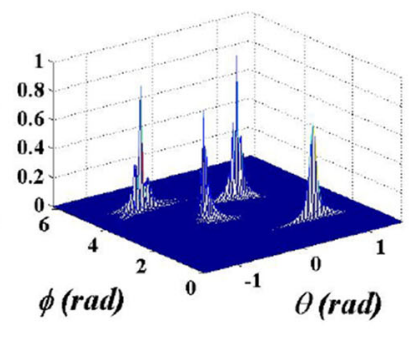

c

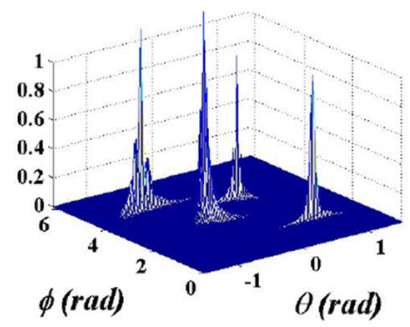

g

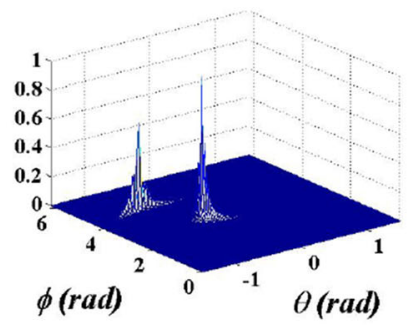

d

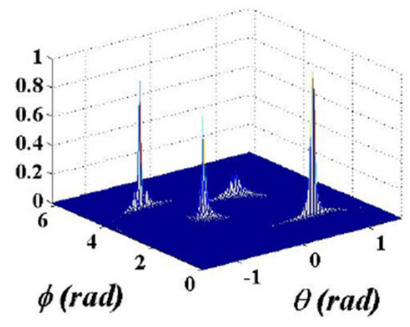

h

Fig. 1 Direction finding with four sources in the presence of noncoherent noise: a direction finding using the LV-MUSIC estimator when $\gamma=\pi / 4, \eta=-\pi / 2$, b direction finding using the DOA-LV-MUSIC estimator, c direction finding using the DOA-P-BB-MUSIC estimator, $\mathbf{d}$ direction finding using the DOA-BB-MUSIC estimator when $\gamma \neq \pi / 4, \eta \neq-\pi / 2$, e direction finding using the DOA-BB-MUSIC estimator when $\gamma=\pi / 4, \eta=-\pi / 2$, f direction finding using the DOA-BB-MUSIC estimator when $\gamma=\pi / 4, \eta=-\pi / 2$, $\mathbf{g}$ the sum of the spectrums in $\mathbf{d}-\mathbf{f}$, and $\mathbf{h}$ direction finding using the QQ-MUSIC estimator 
The second part of the simulation will verify the validity of the DOA-PB-BB-MUSIC algorithm, and then compares the results with the DOA-LV-MUSIC and DOABB-MUSIC respectively. We consider an array of seven vector-sensors and two uncorrelated narrow-band incident sources with the following simulation parameters:

$$
\begin{aligned}
& \phi_{1}=2.8 \mathrm{rad}, \theta_{1}=0.1 \mathrm{rad}, \gamma_{1}=0.7 \mathrm{rad}, \eta_{1}=0.3 \mathrm{rad} \\
& \phi_{2}=2.4 \mathrm{rad}, \theta_{2}=0.6 \mathrm{rad}, \gamma_{2}=0.1 \mathrm{rad}, \eta_{2}=-1.5 \mathrm{rad}
\end{aligned}
$$

Assume that the noises of the vector-sensors are uncorrelated, while the noises among the three electric and magnetic antennas in a vector sensor are correlated. With an SNR $=-15 \mathrm{~dB}$ and 2000 snapshots is used, the simulation results are shown in Fig. 2.

It can easy find from Fig. 2 that the correlated noise in each vector sensor has relatively greater effect on the performances of DOA-LV-MUSIC and DOA-BB-MUSIC algorithms when the SNR is lower. The performance of the DOA-PB-BB-MUSIC algorithm is greatly improved since the constructed beamspace has whitened the coherent noise.

The third simulation tests robustness of the direction finding performances of the estimators to corrupting noise. Two sources with $\left\{\phi_{1}=2.8 \mathrm{rad}, \theta_{1}=0.1 \mathrm{rad}, \gamma_{1}=\right.$ $\left.0.7 \mathrm{rad}, \eta_{1}=0.3 \mathrm{rad}\right\}$ and $\left\{\phi_{2}=2.4 \mathrm{rad}, \theta_{2}=0.6 \mathrm{rad}, \gamma_{2}=\right.$ $\left.0.1 \mathrm{rad}, \eta_{2}=-1.5 \mathrm{rad}\right\}$ are taken. The number of vectorsensors in the array is 7 . For each point on Fig. 3, the simulations with 300 independent Monte Carlo experiments are run. Figure $3 \mathrm{a}$ plots the composite RMS errors of the direction finding versus the different SNRs in the presence of noncoherent noise. Figure $3 \mathrm{~b}$ illustrates the behaviors of the different estimators in the presence of coherent noise. When the corrupting noise is noncoherent, the performances of the DOA-LV-MUSIC are the best while the DOA-BB-MUSIC and ESPRIT estimators are better than the DOA-PB-BB-MUSIC one as shown in Fig. 3a. However, in the coherent noise case, the performances of the DOA-BB-MUSIC and DOA-PB-BB-MUSIC are much better than those of the DOA-LV-MUSIC and ESPRIT ones as depicted in Fig. 3b. Seeing from the $y$-axes of Fig. 3, one can find that the performances of the DOA-PB-BBMUSIC yield almost same performances in both coherent and noncoherent corrupting noise situations. As a contrast, the composite RMS errors of the DOA-LV-MUSIC in the coherent case is almost 10 times larger than those in the noncoherent case with low SNRs, while the RMS errors of the ESPRIT in the coherent case is five times larger than those in the noncoherent case with low SNRs. As a result, the performances of the DOA-LV-MUSIC and ESPRIT are strongly degraded by the coherent noise. To evaluate the performances of the DOA-LV-MUSIC and ESPRIT after the correlation of noise is whiten, we assume that the noise covariance matrix can be estimated and then is used to whiten the received signal covariance matrix as done in [26]. It is understood by means of the method in [26] that it is impossible for one to completely whitened the correlated noise due to the estimation errors of the noise covariance matrix. Thus, it is assumed that the noise correlation has dropped to one tenth of the original after whitening. Figure $3 \mathrm{c}$ illustrates the performances of the ESPRIT and standard MUSIC estimators after whitening the coherent noise and the DOA-PB-BBMUSIC estimator in the presence of coherent noise. Even so, it can be seen that the performances of the DOA-PBBB-MUSIC estimator is still better than the ESPRIT and standard MUSIC estimators. It also verifies that our estimators are more robust to coherent corrupting noise than the known ones.

Using the DOA-PB-BB-MUSIC estimator, we will next test its robustness to the polarization parameters. The same array and source configuration as the second simulation is taken into consideration.

Since the DOA-BB-MUSIC and QQ-MUSIC estimators cannot estimate the polarization angles, we only calculate the composite RMS errors of the polarization estimations of the DOA-PB-BB-MUSIC and ESPRIT estimators with different SNRs under both the noncoherent and coherent noise conditions.

Based on the direction finding results in the second simulation in each Monte Carlo run, the algorithm via (36) is used to estimate the polarization parameters. From Fig. 4a

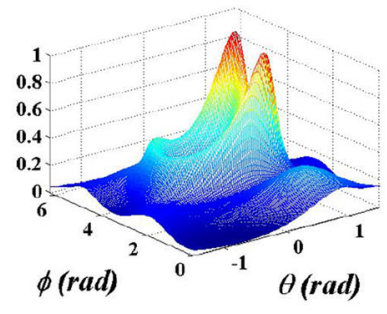

a

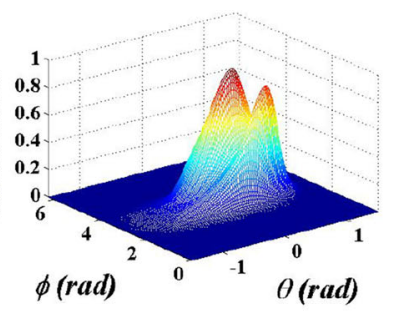

b

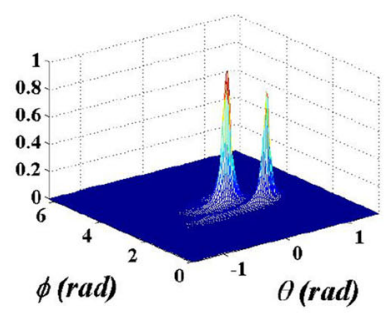

c

Fig. 2 Direction finding with two sources in the presence of coherent noise: a DOA-LV-MUSIC, b DOA-BB-MUSIC, and c DOA-PB-BB-MUSIC 


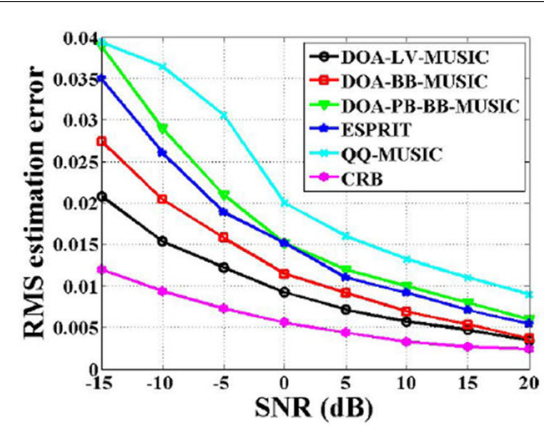

a

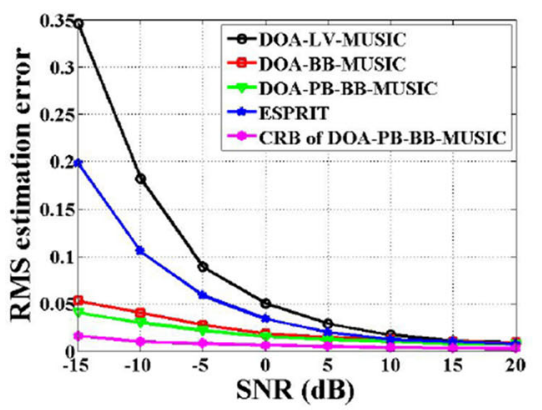

b

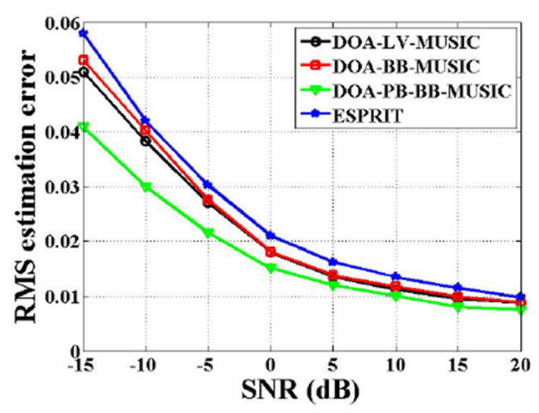

c

Fig. 3 The composite RMS estimation errors of direction finding versus SNR: a The composite RMS estimation errors of direction finding in presence of noncoherent noise, and $\mathbf{b}$ The composite RMS estimation errors of direction finding in presence of coherent noise, and $\mathbf{c}$ The composite RMS estimation errors of direction finding after whitening the coherent noise

and $b$, we find that the performances of the ESPRIT estimators in the coherent case are obviously degraded. It can also be seen that its composite RMS errors in Fig. 4b, are almost 4 times larger than those in Fig. 4a. Contrastively, the composite RMS errors of our DOA-PB-BB-MUSIC estimator are almost the same for both cases, illustrating the robustness of our estimator to corrupting coherent noise again.

\section{Conclusions}

In this paper, we have illustrated the methods of electromagnetic vector-sensor array signal processing in our biquaternion beamspace. In our biquaternion beamspace, the memory spaces for storing the data covariance matrix and the computation efforts for performing its EVD are reduced. Techniques for choosing the transformation matrix matrices to blindly whiten the coherence of the electric and magnetic noise in a vector-sensor have been given. These techniques ensure that our BBMUSIC estimator can be extended to a certain coherent noise case and is more robust to general coherent noise cases. Three enhanced versions of BB-MUSIC estimator are also presented. The three estimators are capable of decoupling polarization estimation from direction finding. In addition, we did not solve the optimization problem (37) in theory, instead we use the numerical solution. The optimal solution will be considered in our future work.

As an alternative way for vector-sensor array processing, by choosing proper transformation matrix, the biquaternion beamspace theory presented here may be applied to other cases, such as enhancing the signals from favored directions and block interferences from other directions. These topics will also be the focus of our future work.

\section{Endnotes}

${ }^{1}$ In order not to confuse the imaginary unit of a complex number with the three imaginary units of a quaternion, we use the notations in [13] that $I$ is the imaginary unit

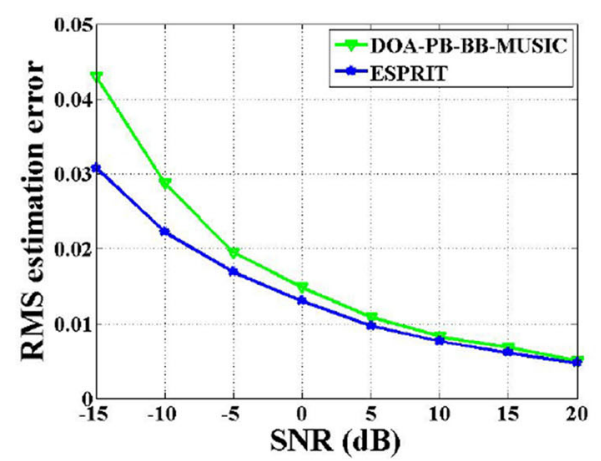

a

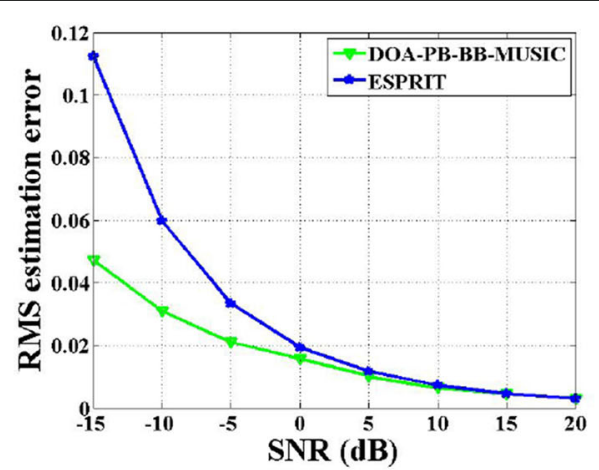

b

Fig. 4 The composite RMS estimation errors of polarization estimation versus SNR: a the composite RMS estimation errors of polarization estimation in presence of noncoherent noise and $\mathbf{b}$ the composite RMS estimation errors of polarization estimation in presence of coherent noise 
of a complex number while $i, j$ and $k$ denote the three imaginary units of a quaternion.

${ }^{2}$ In [13], the EVD of a biquaternion matrix is obtained from the EVD of its quaternion adjoint matrix. Alternatively, the EVD of a biquaternion matrix can also be achieved by the EVD of its complex representation matrix [20].

${ }^{3}$ This can be get directly from the EVD of the quaternion adjoint matrix of $\boldsymbol{R}_{z}(t)$ [22] and the Q-MUSIC estimator in [11], or the EVD of the complex representation matrix of $\boldsymbol{R}_{z}(t)$ and the traditional MUSIC estimator [2,3].

${ }^{4}$ The third and fourth sources are well known as the leftcircularly polarization and right-circularly polarization sources respectively.

${ }^{5}$ If there are some sources happen to have same direction angles but different polarization angles, the polarization decoupled MUSIC like estimators may fail to discriminate these sources because only a $2 \mathrm{D}$ search over $\left(\theta_{k}, \phi_{k}\right)$ is performed.

\section{Appendix 1: Proof of Theorem 1}

Since

$v_{k 1}=\boldsymbol{t}_{a} v_{1}=-\sin \phi_{k} e_{1}+\cos \phi_{k} e_{2}$

$v_{k 2}=\boldsymbol{t}_{a} v_{2}=-\cos \phi_{k} \sin \theta_{k} e_{1}-\sin \phi_{k} \sin \theta_{k} e_{2}+\cos \theta_{k} e_{3}$,

we have

$u_{k}=v_{k 1} v_{k 2}=\sin \theta_{k} e_{1} e_{2}-\sin \phi_{k} \cos \theta_{k} e_{1} e_{3}+\cos \phi_{k} \cos \theta_{k} e_{2} e_{3}$

and

$v_{k 1} v_{k 1}=\sin \phi_{k}^{2} e_{1} e_{1}+\cos \phi_{k}^{2} e_{2} e_{2}-\sin \phi_{k} \cos \phi_{k}\left(e_{1} e_{2}+e_{2} e_{1}\right)$,

where $e_{1}, e_{2}, e_{3}$ are unit quaternions orthogonal to one another. Since $e_{1} e_{2}+e_{2} e_{1}=0$ and $e_{1} e_{1}=e_{2} e_{2}=e_{3} e_{3}=$ $-1 / 3, v_{k 1} v_{k 1}=-1 / 3$. Similarly we can also get $v_{k 2} v_{k 2}=$ $-1 / 3$.

In the same manner, we have

$$
v_{k 2} v_{k 1}=-v_{k 1} v_{k 2}
$$

As a result, the following equations can be deduced:

$$
\begin{aligned}
u_{k} u_{k} & =v_{k 1} v_{k 2} v_{k 1} v_{k 2}=v_{k 1}\left(-v_{k 1} v_{k 2}\right) v_{k 2}=-1 / 9 \\
u_{k} v_{k 1} & =v_{k 1} v_{k 2} v_{k 1}=\left(-v_{k 2} v_{k 1}\right) v_{k 1}=1 / 3 v_{k 2} \\
u_{k} v_{k 2} & =v_{k 1} v_{k 2} v_{k 2}=-1 / 3 v_{k 1}
\end{aligned}
$$

From (12) we know

$$
\left(\boldsymbol{T} \boldsymbol{a}_{k}\right)^{\dagger} \boldsymbol{E}_{n}=\mathbf{0}_{4 M-2 K},(k=1,2, \cdots K)
$$

where

$$
\begin{aligned}
\boldsymbol{T a}_{k} & =\boldsymbol{I}_{M} \otimes\left[\begin{array}{cc}
\boldsymbol{t}_{a} & 0 \\
0 & \boldsymbol{t}_{b}
\end{array}\right]\left(\boldsymbol{q}\left(\theta_{k}, \phi_{k}\right) \otimes \boldsymbol{V}\left(\theta_{k}, \phi_{k}\right) \boldsymbol{P}\left(\gamma_{k}, \eta_{k}\right)\right) \\
& =\boldsymbol{q}\left(\theta_{k}, \phi_{k}\right) \otimes\left[\begin{array}{cc}
v_{k 1} & -v_{k 2} \\
v_{k 2} & v_{k 1}
\end{array}\right] \boldsymbol{P}\left(\gamma_{k}, \eta_{k}\right) \\
& =\boldsymbol{q}\left(\theta_{k}, \phi_{k}\right) \otimes\left[\begin{array}{c}
1 \\
3 u_{k}
\end{array}\right]\left(v_{k 1} \cos \gamma_{k}-v_{k 2} \sin \gamma_{k} e^{I \eta_{k}}\right)
\end{aligned}
$$

Let $\boldsymbol{W}=\boldsymbol{q}\left(\theta_{k}, \phi_{k}\right) \otimes\left[\begin{array}{l}1 \\ 3 u_{k}\end{array}\right], \boldsymbol{\Delta}_{k}=\boldsymbol{W}^{\dagger} \boldsymbol{E}_{n}, \rho_{k}=$ $\left(v_{k 1} \cos \gamma_{k}-v_{k 2} \sin \gamma_{k} e^{I \eta_{k}}\right)^{\dagger}$, and then insert them into (12), we have $\rho_{k} \boldsymbol{\Delta}_{k}=\mathbf{0}_{4 M-2 K}$.

Since

$\rho_{k}^{\dagger} \rho_{k}=\left(v_{k 1} \cos \gamma_{k}-v_{k 2} \sin \gamma_{k} e^{I \eta_{k}}\right)\left(v_{k 1} \cos \gamma_{k}-v_{k 2} \sin \gamma_{k} e^{I \eta_{k}}\right)^{\dagger}$ $=1 / 3-I u_{k} \sin 2 \gamma_{k} \sin \eta_{k}$,

we have

$\left(1 / 3+I u_{k} \sin 2 \gamma_{k} \sin \eta_{k}\right) \rho_{k}^{\dagger} \rho_{k}=\frac{1}{9}\left(1-\left(\sin 2 \gamma_{k} \sin \eta_{k}\right)^{2}\right)$.

so

$$
\begin{aligned}
& \left(1 / 3+I u_{k} \sin 2 \gamma_{k} \sin \eta_{k}\right) \rho_{k}^{\dagger} \rho_{k} \boldsymbol{\Delta}_{k}=\frac{1}{9} \\
& \left(1-\left(\sin 2 \gamma_{k} \sin \eta_{k}\right)^{2}\right) \boldsymbol{\Delta}_{k}=\mathbf{0}_{4 M-2 N} .
\end{aligned}
$$

When $\gamma_{k} \neq \pi / 4, \eta_{k} \neq \pm \pi / 2$, we get $\boldsymbol{\Delta}=\mathbf{0}_{4 M-s K}$. That is:

$$
\left(\boldsymbol{q}\left(\theta_{k}, \phi_{k}\right) \otimes\left[\begin{array}{c}
1 \\
3 u_{k}
\end{array}\right]\right)^{\dagger} \boldsymbol{E}_{n}=\mathbf{0}_{4 M-2 K},(k=1,2, \cdots K)
$$

\section{Appendix 2: Proof of Theorem 2}

Let

$$
\boldsymbol{\Theta}\left(\theta_{k}, \phi_{k}\right)=\boldsymbol{I}_{M} \otimes\left[\begin{array}{cc}
\boldsymbol{t}_{a} & 0 \\
0 & \boldsymbol{t}_{b}
\end{array}\right]\left(\boldsymbol{q}\left(\theta_{k}, \phi_{k}\right) \otimes \boldsymbol{V}\left(\theta_{k}, \phi_{k}\right)\right) .
$$

We have

$$
\begin{aligned}
\boldsymbol{\Theta}\left(\theta_{k}, \phi_{k}\right) & =\boldsymbol{I}_{M} \otimes\left[\begin{array}{cc}
\boldsymbol{t}_{a} & 0 \\
0 & \boldsymbol{t}_{b}
\end{array}\right]\left(\boldsymbol{q}\left(\theta_{k}, \phi_{k}\right) \otimes \boldsymbol{V}\left(\theta_{k}, \phi_{k}\right)\right) \\
& =\boldsymbol{I}_{M} \boldsymbol{q}\left(\theta_{k}, \phi_{k}\right) \otimes\left(\left[\begin{array}{cc}
\boldsymbol{t}_{a} & 0 \\
0 & \boldsymbol{t}_{b}
\end{array}\right] \boldsymbol{V}\left(\theta_{k}, \phi_{k}\right)\right) \\
& =\left(\boldsymbol{q}\left(\theta_{k}, \phi_{k}\right) \otimes \boldsymbol{I}_{2}\right)\left[\begin{array}{cc}
v_{k 1} & -v_{k 2} \\
v_{k 2} & v_{k 1}
\end{array}\right] .
\end{aligned}
$$

If $\boldsymbol{Q}\left(\theta_{k}, \phi_{k}\right)=\boldsymbol{q}\left(\theta_{k}, \phi_{k}\right) \otimes \boldsymbol{I}_{2}$, then $\boldsymbol{\Theta}\left(\theta_{k}, \phi_{k}\right)$ can be rewritten as

$$
\boldsymbol{\Theta}\left(\theta_{k}, \phi_{k}\right)=\boldsymbol{Q}\left(\theta_{k}, \phi_{k}\right)\left[\begin{array}{cc}
v_{k 1} & -v_{k 2} \\
v_{k 2} & v_{k 1}
\end{array}\right]
$$

From (15), it has known that, if $\boldsymbol{\Lambda}_{k}=$ $\left[\boldsymbol{\Theta}\left(\theta_{k}, \phi_{k}\right)\right]^{\dagger} \boldsymbol{E}_{n} \boldsymbol{E}_{n}^{\dagger}\left[\boldsymbol{\Theta}\left(\theta_{k}, \phi_{k}\right)\right]$, one has $\boldsymbol{\Xi}\left(\theta_{k}, \phi_{k}\right)=$ 
$\mathbb{S}\left\{\boldsymbol{\Lambda}_{k}\right\}$, where

$$
\begin{aligned}
\boldsymbol{\Lambda}_{k} & =\left[\boldsymbol{\Theta}\left(\theta_{k}, \phi_{k}\right)\right]^{\dagger} \boldsymbol{E}_{n} \boldsymbol{E}_{n}^{\dagger}\left[\boldsymbol{\Theta}\left(\theta_{k}, \phi_{k}\right)\right] \\
& =\left[\begin{array}{cc}
v_{k 1} & -v_{k 2} \\
v_{k 2} & v_{k 1}^{\dagger}
\end{array}\right]^{\dagger} \boldsymbol{Q}\left(\theta_{k}, \phi_{k}\right)^{\dagger} \boldsymbol{E}_{n} \boldsymbol{E}_{n}^{\dagger} \boldsymbol{Q}\left(\theta_{k}, \phi_{k}\right)\left[\begin{array}{cc}
v_{k 1} & -v_{k 2} \\
v_{k 2} & v_{k 1}
\end{array}\right] .
\end{aligned}
$$

Let $\boldsymbol{X}=\boldsymbol{Q}^{H}\left(\theta_{k}, \phi_{k}\right) \boldsymbol{E}_{n} \boldsymbol{E}_{n}^{\dagger} \boldsymbol{Q}\left(\theta_{k}, \phi_{k}\right)$. Based on $\boldsymbol{X} \in \mathbb{H}_{\mathbb{C}}^{2 \times 2}$ and $\boldsymbol{X}=\boldsymbol{X}^{\dagger}, \boldsymbol{X}$ can be expressed as follows:

$$
\boldsymbol{X}=\left[\begin{array}{cc}
x_{10}+I x_{1 q} & x_{30}^{H}+x_{3 q}^{\dagger} \\
x_{30}+x_{3 q} & x_{20}+I x_{2 q}
\end{array}\right]
$$

where $x_{10}$ and $x_{20}$ are real numbers, $x_{30}$ is a complex number, $x_{1 q}$ and $x_{2 q}$ are pure quaternions, $x_{3 q}$ is a pure biquaternion. Insert (40) into (39), we have

$$
\begin{aligned}
\boldsymbol{\Lambda}_{k} & =\left[\begin{array}{cc}
v_{k 1} & -v_{k 2} \\
v_{k 2} & v_{k 1}
\end{array}\right]^{\dagger} \boldsymbol{X}\left[\begin{array}{cc}
v_{k 1} & -v_{k 2} \\
v_{k 2} & v_{k 1}
\end{array}\right] \\
& =\left[v_{k 1}-v_{k 2}\right]^{\dagger}\left[\begin{array}{l}
1 \\
3 u_{k}
\end{array}\right]^{\dagger}\left[\begin{array}{cc}
x_{10}+I x_{1 q} & x_{30}^{H}+x_{3 q}^{\dagger} \\
x_{30}+x_{3 q} & x_{20}+I x_{2 q}
\end{array}\right]\left[\begin{array}{l}
1 \\
3 u_{k}
\end{array}\right]\left[v_{k 1}-v_{k 2}\right] .
\end{aligned}
$$

Since $v_{k 1}, v_{k 2}$ and $u_{k}$ are pure quaternions, we can get $v_{k 1}{ }^{\dagger}=v_{k 1} \triangleleft=-v_{k 1}, v_{k 2}{ }^{\dagger}=v_{k 2}{ }^{\triangleleft}=-v_{k 2}$ and $u_{k}^{\dagger}=$ $u_{k}{ }^{\triangleleft}=-u_{k}$. In this way, (39) can be rewritten as:

$$
\begin{aligned}
\boldsymbol{\Lambda}_{k}= & {\left[\begin{array}{l}
-v_{k 1} \\
v_{k 2}
\end{array}\right]\left(x_{10}+x_{20}+I x_{1 q}-3 x_{30} u_{k}+3 x_{30}^{H} u_{k}\right.} \\
& \left.-3 u_{k} x_{3 q}+3 x_{3 q}^{\dagger} u_{k}-I 9 u_{k} x_{2 q} u_{k}\right)\left[v_{k 1}-v_{k 2}\right] .
\end{aligned}
$$

Let $\boldsymbol{\Lambda}_{k}=\left[\begin{array}{ll}\boldsymbol{\Lambda}_{k 11} & \boldsymbol{\Lambda}_{k 12} \\ \boldsymbol{\Lambda}_{k 21} & \boldsymbol{\Lambda}_{k 22}\end{array}\right]$, then $\boldsymbol{\Xi}\left(\theta_{k}, \phi_{k}\right)=\mathbb{S}\left\{\boldsymbol{\Lambda}_{k}\right\}=$ $\left[\begin{array}{ll}\mathbb{S}\left(\boldsymbol{\Lambda}_{k 11}\right) & \mathbb{S}\left(\boldsymbol{\Lambda}_{k 12}\right) \\ \mathbb{S}\left(\boldsymbol{\Lambda}_{k 21}\right) & \mathbb{S}\left(\boldsymbol{\Lambda}_{k 22}\right)\end{array}\right]$. Notice that as far as any two arbitrary pure quaternions $q_{1}$ and $q_{2}$ are concerned, we know $\mathbb{S}\left\{q_{1} q_{2} q_{1}\right\}=\mathbb{S}\left\{q_{2} q_{1} q_{2}\right\}=0$, where $\mathbb{S}(\cdot)$ stands for taking the scalar part of the biquaternion. In other words, $q_{1} q_{2} q_{1}$ and $q_{2} q_{1} q_{2}$ are pure quaternions. Since all of $v_{k 1}, v_{k 2}, u_{k}, x_{1 q}$ and $x_{2 q}$ are pure quaternions, $u_{k} x_{2 q} u_{k}$ is also a pure quaternion. When $q_{b 1}=v_{k 2} x_{3 q} v_{k 1}$ and $q_{b 2}=$ $v_{k 1} x_{3 q}{ }^{\dagger v_{k 2}}$, we can get

$$
\mathbb{S}\left(\boldsymbol{\Lambda}_{k 11}\right)=1 / 3 x_{10}+1 / 3 x_{20}-\mathbb{S}\left(q_{b 1}\right)-\mathbb{S}\left(q_{b 2}\right) .
$$

It can be seen that $q_{b 1}{ }^{\dagger}=v_{k 1}{ }^{\dagger} x_{3 q}^{\dagger} v_{k 2}{ }^{\dagger}=v_{k 1} x_{3 q}^{\dagger} v_{k 2}=$ $q_{b 2}$. As a result, one has $\mathbb{S}\left(q_{b 1}\right)=\mathbb{S}\left(q_{b 2}\right)^{*}$. That is $\mathbb{S}\left(q_{b 1}\right)+$ $\mathbb{S}\left(q_{b 2}\right) \in \mathbb{R}$. Since both $x_{10}$ and $x_{20}$ are real numbers, we have $\mathbb{S}\left(\boldsymbol{\Lambda}_{k 11}\right)=1 / 3 x_{10}+1 / 3 x_{20}-\left(\mathbb{S}\left(q_{b 1}\right)+\mathbb{S}\left(q_{b 2}\right)\right) \in \mathbb{R}$ and then let $\mathbb{S}\left(\boldsymbol{\Lambda}_{k 11}\right)=a$.

Similarly, since $\mathbb{S}\left(q_{b 3}\right)+\mathbb{S}\left(q_{b 4}\right)=-2 \mathbb{S}\left(v_{k 2} x_{3 q 1} v_{k 1}\right)$, it can be deduced that

$$
\begin{aligned}
& \mathbb{S}\left(q_{b 3}\right)+\mathbb{S}\left(q_{b 4}\right)=-\left(\mathbb{S}\left(q_{b 1}\right)+\mathbb{S}\left(q_{b 2}\right)\right) \\
& \text { Therefore } \mathbb{S}\left(\boldsymbol{\Lambda}_{k 22}\right)=\mathbb{S}\left(\boldsymbol{\Lambda}_{k 11}\right)=a .
\end{aligned}
$$

From (42), we have

$$
\begin{aligned}
\boldsymbol{\Lambda}_{k 12}= & x_{10} v_{k 1} v_{k 2}+I v_{k 1} x_{1 q} v_{k 2}-1 / 3 x_{30}+1 / 3 x_{30}^{H} \\
& +v_{k 2} x_{3 q} v_{k 2}-v_{k 1} x_{3 q}^{\dagger} v_{k 1}+x_{20} v_{k 1} v_{k 2}-I v_{k 2} x_{2 q} v_{k 1} .
\end{aligned}
$$

Thus, we can know $\mathbb{S}\left(\boldsymbol{\Lambda}_{k 12}\right)=1 / 3\left(x_{30}-x_{30}^{H}\right)+$ $\mathbb{S}\left(I v_{k 1} x_{1 q} v_{k 2}\right)+\mathbb{S}\left(I v_{k 2} x_{2 q} v_{k 1}\right)$. Apparently $\boldsymbol{\Lambda}_{k 12}$ is a pure imaginary number. In this way, we can make $\boldsymbol{\Lambda}_{k 12}=-b I$, where $b$ is a real number.

Similarly let $\mathbb{S}\left(\boldsymbol{\Lambda}_{k 12}\right)=1 / 3 x_{30}-1 / 3 x_{30}^{H}+$ $\mathbb{S}\left(I v_{k 2} x_{1 q} v_{k 1}\right)+\mathbb{S}\left(I v_{k 1} x_{2 q} v_{k 2}\right)=b I$, so we can get $\boldsymbol{\Xi}\left(\theta_{k}, \phi_{k}\right)=\mathbb{S}\left\{\boldsymbol{\Lambda}_{k}\right\}=\left[\begin{array}{ll}\mathbb{S}\left(\boldsymbol{\Lambda}_{k 11}\right) & \mathbb{S}\left(\boldsymbol{\Lambda}_{k 12}\right) \\ \mathbb{S}\left(\boldsymbol{\Lambda}_{k 21}\right) & \mathbb{S}\left(\boldsymbol{\Lambda}_{k 22}\right)\end{array}\right]=\left[\begin{array}{cc}a & -b I \\ b I & a\end{array}\right]$.

\section{Appendix 3}

According to (40), $\boldsymbol{\Lambda}_{k}$ can be changed into

$$
\begin{aligned}
\boldsymbol{\Lambda}_{k} & =\left[\begin{array}{cc}
t_{a} v_{1} & -t_{a} v_{2} \\
t_{b} v_{2} & t_{b} v_{1}
\end{array}\right]^{\dagger} \boldsymbol{X}\left[\begin{array}{cc}
t_{a} v_{1} & -t_{a} v_{2} \\
t_{b} v_{2} & t_{b} v_{1}
\end{array}\right] \\
& =\left[\begin{array}{cc}
t_{a} v_{1} & -t_{a} v_{2} \\
t_{b} v_{2} & t_{b} v_{1}
\end{array}\right]^{\dagger}\left[\begin{array}{cc}
x_{10}+I x_{1 q} & x_{30}^{H}+x_{3 q}^{\dagger} \\
x_{30}+x_{3 q} & x_{20}+I x_{2 q}
\end{array}\right]\left[\begin{array}{cc}
t_{a} v_{1} & -t_{a} v_{2} \\
t_{b} v_{2} & t_{b} v_{1}
\end{array}\right]
\end{aligned}
$$

Suppose that $v_{k 1}=t_{a} v_{1}, v_{k 2}=-t_{a} v_{2}, v_{k 3}=t_{a} v_{2}, v_{k 4}=$ $t_{a} v_{1}$, all of $v_{k 1}, v_{k 2}, v_{k 3}, v_{k 4}$ are pure biquaternions. One can get

$$
\begin{aligned}
\Lambda_{k 11}= & -\left(v_{k 1} x_{10} v_{k 1}+I v_{k 1} x_{1 q} v_{k 1}+v_{k 3} x_{30} v_{k 1}\right. \\
& +v_{k 3} x_{3 q} v_{k 1}+v_{k 1} x_{30}^{H} v_{k 3}+v_{k 1} x_{3 q}^{\dagger} v_{k 3} \\
& \left.+v_{k 3} x_{20} v_{k 3}+I v_{k 3} x_{2 q} v_{k 3}\right)
\end{aligned}
$$

and

$$
\begin{aligned}
\mathbb{S}\left(\boldsymbol{\Lambda}_{k 11}\right) & =1 / 3 x_{10}+1 / 3 x_{20}-\left(x_{30}+x_{30}^{H}\right) \mathbb{S}\left(v_{k 1} v_{k 3}\right) \\
& -\left(\mathbb{S}\left(v_{k 3} x_{3 q} v_{k 1}\right)+\mathbb{S}\left(v_{k 1} x_{3 q}^{\dagger} v_{k 3}\right)\right)
\end{aligned}
$$

Since $\mathbb{S}\left(v_{k 3} x_{3 q} v_{k 1}\right)+\mathbb{S}\left(v_{k 1} x_{3 q}^{\dagger} v_{k 3}\right)$ is real, $\mathbb{S}\left(\boldsymbol{\Lambda}_{k 11}\right) \in \mathbb{R}$. Thus, we can let $\mathbb{S}\left(\boldsymbol{\Lambda}_{k 11}\right)=a_{\Lambda 1} \in \mathbb{R}$. Similarly,

$$
\begin{aligned}
\mathbb{S}\left(\boldsymbol{\Lambda}_{k 22}\right) & =1 / 3 x_{10}+1 / 3 x_{20}-\left(x_{30}+x_{30}^{H}\right) \mathbb{S}\left(v_{k 2} v_{k 4}\right) \\
& -\left(\mathbb{S}\left(v_{k 4} x_{3 q} v_{k 2}\right)+\mathbb{S}\left(v_{k 2} x_{3 q}^{\dagger} v_{k 4}\right)\right) \in \mathbb{R} .
\end{aligned}
$$

Additionally, $\mathbb{S}\left(\boldsymbol{\Lambda}_{k 12}\right)$ can be written as

$$
\begin{aligned}
\mathbb{S}\left(\boldsymbol{\Lambda}_{k 12}\right)= & -I \mathbb{S}\left(v_{k 1} x_{1 q} v_{k 2}\right)-I \mathbb{S}\left(v_{k 3} x_{2 q} v_{k 4}\right) \\
& -x_{30} \mathbb{S}\left(v_{k 3} v_{k 2}\right)-x_{30}^{H} \mathbb{S}\left(v_{k 1} v_{k 4}\right) \\
& -\mathbb{S}\left(v_{k 3} x_{3 q} v_{k 2}\right)-\mathbb{S}\left(v_{k 1} x_{3 q}^{\dagger} v_{k 4}\right) .
\end{aligned}
$$


Since $\mathbb{S}\left(\boldsymbol{\Lambda}_{k 12}\right)$ is a complex number, one can let $\mathbb{S}\left(\boldsymbol{\Lambda}_{k 12}\right)=a_{\Lambda 12}+I b_{\Lambda 12}$. It is easy to find out that

$$
\begin{aligned}
\mathbb{S}\left(\boldsymbol{\Lambda}_{k 21}\right)= & -I \mathbb{S}\left(v_{k 2} x_{1 q} v_{k 1}\right)-I \mathbb{S}\left(v_{k 4} x_{2 q} v_{k 3}\right) \\
& -x_{30} \mathbb{S}\left(v_{k 4} v_{k 1}\right)-x_{30}^{H} \mathbb{S}\left(v_{k 2} v_{k 3}\right) \\
& -\mathbb{S}\left(v_{k 4} x_{3 q} v_{k 1}\right)-\mathbb{S}\left(v_{k 2} x_{3 q}^{\dagger} v_{k 3}\right)
\end{aligned}
$$

Due to $\left(\mathbb{S}\left(\boldsymbol{\Lambda}_{k 12}\right)\right)^{*}=\mathbb{S}\left(\boldsymbol{\Lambda}_{k 21}\right)$ and taking $\mathbb{S}\left(\boldsymbol{\Lambda}_{k 21}\right)=$ $a_{\Lambda 12}-I b_{\Lambda 12}$, we have

$$
\Xi\left(\theta_{k}, \phi_{k}\right)=\mathbb{S}\left\{\boldsymbol{\Lambda}_{k}\right\}=\left[\begin{array}{cc}
a_{\Lambda 1} & a_{\Lambda 12}+I b_{\Lambda 12} \\
a_{\Lambda 12}-I b_{\Lambda 12} & a_{\Lambda 2}
\end{array}\right]
$$

it can be seen that the $a_{\Lambda 1}, a_{\Lambda 2}, a_{\Lambda 12}$ and $b_{\Lambda 12}$ in (44) are only related to DOA parameters. Insert (44) into (14), and we have

$$
\begin{aligned}
\mathcal{F}\left(\Omega_{k}\right)= & 1 /\left(a_{\Lambda 1} \cos \gamma_{k}^{2}+a_{\Lambda 2} \sin \gamma_{k}^{2}\right. \\
& \left.+a_{\Lambda 12} \sin 2 \gamma_{k} \cos \eta_{k}-b_{\Lambda 12} \sin 2 \gamma_{k} \sin \eta_{k}\right)
\end{aligned}
$$

\section{Appendix 4}

Refer to (3.4) in [4], the CRB on the covariance matrix of any (locally) unbiased estimator of the vector sensor array in the presence of the noncoherent corrupting noise is

$$
\mathrm{CRB}(\Omega)_{l v}=\frac{\sigma^{2}}{2 L}\left\{\operatorname{Re}\left[\operatorname{btr}\left((\mathbf{1} \otimes \boldsymbol{U}) \odot\left(\boldsymbol{D}^{H} \boldsymbol{\Pi}_{c} \boldsymbol{D}\right)^{b T}\right)\right]\right\}^{-1}
$$

where

$$
\begin{aligned}
& \boldsymbol{U}=\boldsymbol{P}_{s}\left(\boldsymbol{A}^{H} \boldsymbol{A} \boldsymbol{P}_{s}+\sigma^{2} \boldsymbol{I}\right)^{-1} \boldsymbol{A}^{H} \boldsymbol{A} \boldsymbol{P}_{s} \\
& \boldsymbol{\Pi}_{c}=\boldsymbol{E}_{n l v} \boldsymbol{E}_{n l v}{ }^{H} \\
& \boldsymbol{D}=\left[\boldsymbol{D}_{1}^{(1)} \cdots \boldsymbol{D}_{q 1}^{(1)} \cdots \boldsymbol{D}_{1}^{(n)} \cdots \boldsymbol{D}_{q n}^{(n)}\right] \\
& \boldsymbol{D}_{l}^{(k)}=\frac{\partial \boldsymbol{a}_{k}}{\partial \Omega_{l}^{(k)}}
\end{aligned}
$$

where $\boldsymbol{P}_{s}$ is a matrix of the signal power, $L$ denotes snapshots, $\boldsymbol{E}_{n l v}$ are the eigenvectors corresponding to the noise subspaces in the LV-MODEL. We use a orthogonal quaternion transformation matrix $\boldsymbol{T}$ to project the original array data into the biquaternion beamspace, So one has

$$
\begin{gathered}
\boldsymbol{A}_{b q}=\boldsymbol{T} \boldsymbol{A} \\
\boldsymbol{E}_{n}=\boldsymbol{T} \boldsymbol{E}_{n l v}
\end{gathered}
$$

Since the coherent noise of the six constituent antennas in each vector-sensor has been whitened by the matrix
$T$, the CRB of the DOA-PB-BB-MUSIC algorithm in the presence of the coherent corrupting noise can be given by

$$
\operatorname{CRB}(\Omega)=\frac{\sigma^{2}}{2 L}\left\{\operatorname{Re}\left[\operatorname{btr}\left(\left(\mathbf{1} \otimes \boldsymbol{U}_{b q}\right) \odot\left(\boldsymbol{D}_{b q}^{\dagger} \boldsymbol{\Pi}_{c b q} \boldsymbol{D}_{b q}\right)^{b T}\right)\right]\right\}^{-1}
$$

where

$$
\begin{aligned}
& \boldsymbol{U}_{b q}=\boldsymbol{P}_{s}\left(\boldsymbol{A}_{b q}{ }^{\dagger} \boldsymbol{A}_{b q} \boldsymbol{P}_{s}+\sigma^{2} \boldsymbol{I}\right)^{-1} \boldsymbol{A}_{b q}^{\dagger} \boldsymbol{A}_{b q} \boldsymbol{P}_{s} \\
& \boldsymbol{\Pi}_{c b q}=\boldsymbol{E}_{n} \boldsymbol{E}_{n}^{\dagger}
\end{aligned}
$$

$$
D_{b q}=T D
$$

Inserting (50), and (51) into (53), (54), and (55) respectively, one has

$$
\boldsymbol{U}_{b q}=\boldsymbol{P}_{s}\left(\boldsymbol{A}^{H} \boldsymbol{T}^{\triangleleft} \boldsymbol{T} \boldsymbol{A} \boldsymbol{P}_{s}+\sigma^{2} \boldsymbol{I}\right)^{-1} \boldsymbol{A}^{H} \boldsymbol{T}^{\triangleleft} \boldsymbol{T} \boldsymbol{A} \boldsymbol{P}_{s}
$$

$$
\boldsymbol{D}_{b q}^{\dagger} \boldsymbol{\Pi}_{c b q} \boldsymbol{D}_{b q}=\boldsymbol{D}^{H} \boldsymbol{T}^{\triangleleft} \boldsymbol{T} \boldsymbol{E}_{n l v} \boldsymbol{E}_{n l v}{ }^{H} \boldsymbol{T}^{\triangleleft} \boldsymbol{T} \boldsymbol{D}
$$

If the matrix $\boldsymbol{T}$ satisfies $\boldsymbol{T}^{\triangleleft} \boldsymbol{T}=\boldsymbol{I}, \mathrm{CRB}(\Omega)=\operatorname{CRB}(\Omega)_{l v}$.

Funding

This work was supported in part by the NSF of China under Grant 61571131 11604055 .

\section{Authors' contributions}

$\mathrm{DL}$ and JQZ conceived and designed the study. DL, FX, and JFJ performed the experiments. DL and JQZ wrote the paper. DL, FX, JFJ, and JQZ reviewed and edited the manuscript. All authors read and approved the manuscript.

\section{Authors' information}

Dan Li received the the M.S. and Ph.D. degrees in electronic engineering from Fudan University, Shanghai, China, in 2006 and 2013, respectively. He is currently a lecturer at the Department of Electronic Engineering, Fudan University. His research interests include array signal processing and biquaternion with applications in signal processing and image processing. Feng Xu received the Ph.D. degree in electronic engineering from Fudan University, Shanghai, China, in 2010. He is currently an Electronic Engineer at the Department of Electronic Engineering, Fudan University. His research interests include array signal processing and biquaternion with applications in signal processing and image processing.

Jing Fei Jiang received the B.Sc. degree and M.Sc. degrees in electronic engineering from Fudan University, Shanghai, China, in 2008 and 2011 , respectively. His research interests include array signal processing and geometric algebra with applications in signal processing and image processing.

Jian Qiu Zhang received the B.Sc. degree from East of China Institute of Engineering, Nanjing, in 1982, and the M.S. and Ph.D. degrees from Harbin Institute of Technology (HIT), Harbin, China, in 1992 and 1996, respectively. He is currently a Professor with the Department of Electronic Engineering, Fudan University, Shanghai, China. From 1999 to 2002, he was a Senior Research Fellow at the School of Engineering, University of Greenwich, Chatham Maritime, UK In 1998, he was a Visiting Research Scientist at the Institute of Intelligent Power Electronics, Helsinki University of Technology, Espoo, Finland. He was an Associate Professor from 1995 to 1997 and a Lecturer from 1989 to 1994 with the Department of Electrical Engineering, HIT. During 1982 to 1987, he was an Assistant Electronic Engineer at the 544th Factory, Hunan, China. His main research interests are signal processing and its application for advanced sensors, intelligent instrumentation systems and control, and communications. 


\section{Competing interests}

The authors declare that they have no competing interests.

Received: 24 August 2016 Accepted: 6 February 2017

Published online: 15 February 2017

\section{References}

1. JF Bull, Field probe for measuring vector components of an electromagnetic field, 5300885 (1994)

2. H Krim, M Viberg, Two decades of array signal processing research: the parametric approach. IEEE Signal Proc. Mag. 13(4), 67-94 (1996)

3. P Stocia, R Moses, Introduction to Spectral Analysis, 2nd edn. (Upper Saddle River NJ: Prentice-Hall, New Jersey, 2004)

4. A Nehorai, E Paldi, Vector-sensor array processing for electromagnetic source localization. IEEE Trans. Sig. Process. 42(2), 376-398 (1994)

5. KT Wong, MD Zoltowski, Self-initiating music-based direction finding and polarization estimation in spatio-polarizational beamspace. IEEE Trans. Antennas Propag. 48(8), 1235-1245 (2000)

6. J Li, Direction and polarization estimation using arrays with small loops and short dipoles. IEEE Trans. Antennas Propag. 41(3), 379-387 (1993)

7. B Hochwald, A Nehorai, Polarimetric modeling and parameter estimation with applications to remote sensing. IEEE Trans. Sig. Process. 43(8), 1923-1935 (1995)

8. XL Xu, KM Buckley, in International Conference on Acoustics, Speech, and Signal Processing. Statistical performance comparison of music in element-space and beam-space, vol. 4, (1989), pp. 2124-2127

9. HB Lee, M Wengrovitz, Resolution threshold of beamspace music for two closely spaced emitters. IEEE Trans. Acoust. Speech Sig. Process. 38(9), 1545-1559 (1990)

10. GXu, SD Silverstein, RH Roy, T Kailath, Beamspace esprit. IEEE Trans. Sig. Process. 42(2), 349-356 (1994)

11. S Miron, N Le Bihan, Jl Mars, Quaternion-music for vector-sensor array processing. IEEE Trans. Sig. Process. 54(4), 1218-1229 (2006)

12. Z Kunlei, S Wang, F Wang, H Jianguo, Parameter estimation of vector-sensor array in colored noise with quaternion-music. Congr. Image Sig. Process. 5, 483-487 (2008)

13. N Le Bihan, S Miron, Jl Mars, Music algorithm for vector-sensors array using biquaternions. IEEE Trans. Sig. Process. 55(9), 4523-4533 (2007)

14. N Le Bihan, S Miron, JI Mars, High resolution vector-sensor array processing based on biquaternions. IEEE Int. Conf. Acoust. Speech Sig. Process. 4, 1077-1080 (2006)

15. XF Gong, ZW Liu, YG Yu, Direction finding via biquaternion matrix diagonalization with vector-sensors. Sig. Process. 91(4), 821-831 (2011)

16. XF Gong, ZW Liu, YG Yu, Quad-quaternion music for doa estimation using electromagnetic vector sensors. EURASIP J. Advances Sig. Process. 2008(1), 1-14 (2008)

17. JF Jiang, JQ Zhang, Geometric algebra of euclidean 3-space for electromagnetic vector-sensor array processing, part i: Modeling. IEEE Trans. Antennas Propag. 58(12), 3961-3973 (2010)

18. X Gou, Z Liu, Y Yu, X Gong, Capon beamformer for acoustic vector sensor arrays using biquaternions. Int. Conf. Aware. Sci. Technol, 28-31 (2011)

19. X Gou, Z Liu, Y Yu, X Gong, Biquaternion capon beamformer using four-component vector-sensor arrays. Int. J. Sens. Netw. 19(3/4), 171-180 (2015)

20. Y Tian, Matrix theory over the complex quaternion algebra (2000). arXiv preprint math/0004005

21. S Said, N Le Bihan, SJ Sangwine, Fast complexified quaternion fourier transform. IEEE Trans. Sig. Process. 56(4), 1522-1531 (2008)

22. F Zhang, Quaternions and matrices of quaternions. Linear Algebra Appl. 251, 21-57 (1997)

23. KT Wong, MD Zoltowski, Closed-form direction finding and polarization estimation with arbitrarily spaced electromagnetic vector-sensors at unknown locations. IEEE Trans. Antennas Propag. 48(5), 671-681 (2000)

24. JE Ferrara, T Parks, Direction finding with an array of antennas having diverse polarizations. IEEE Trans. Antennas Propag. 31(2), 231-236 (1983)

25. G Strang, Linear Algebra and Its Applications, 3rd edn. (New York Academic, New York, 1976)

26. $\mathrm{PC}$ Hansen, $\mathrm{SH}$ Jensen, Prewhitening for rank-deficient noise in subspace methods for noise reduction. IEEE Trans. Sig. Process. 53(10), 3718-3726 (2005)

\section{Submit your manuscript to a SpringerOpen ${ }^{\circ}$ journal and benefit from:}

- Convenient online submission

- Rigorous peer review

- Immediate publication on acceptance

- Open access: articles freely available online

- High visibility within the field

- Retaining the copyright to your article

Submit your next manuscript at springeropen.com 